Document downloaded from:

http://hdl.handle.net/10251/103293

This paper must be cited as:

Bivià-Ausina, C. (2018). Integral closure and bounds for quotients of multiplicities of monomial ideals. Journal of Algebra. 501:233-254. doi:10.1016/j.jalgebra.2017.12.030

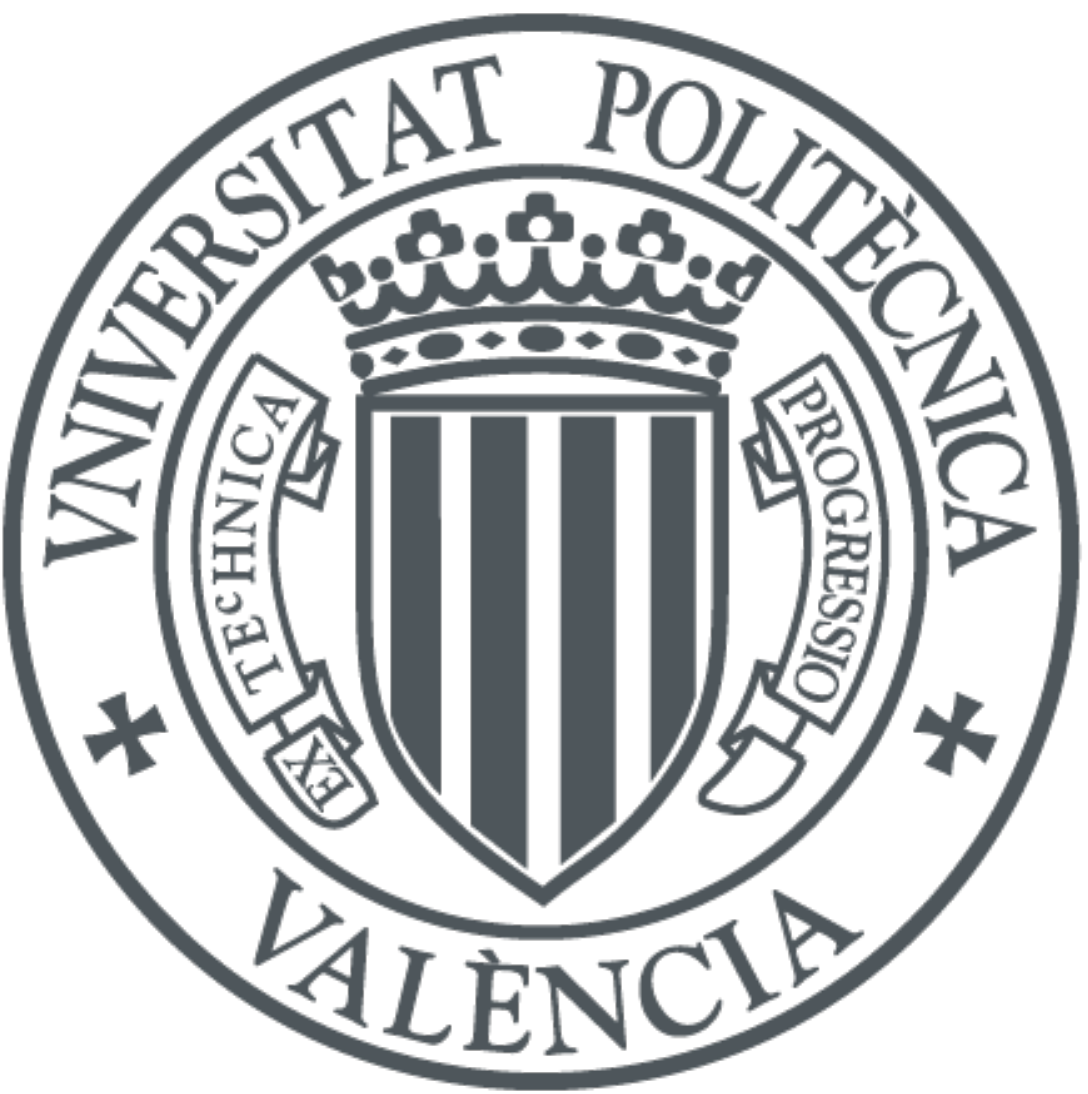

The final publication is available at

https://doi.org/10.1016/j.jalgebra.2017.12.030

Copyright Elsevier

Additional Information 


\title{
INTEGRAL CLOSURE AND BOUNDS FOR QUOTIENTS OF MULTIPLICITIES OF MONOMIAL IDEALS
}

\author{
CARLES BIVIÀ-AUSINA
}

\begin{abstract}
Given a pair of monomial ideals $I$ and $J$ of finite colength of the ring of analytic function germs $\left(\mathbb{C}^{n}, 0\right) \rightarrow \mathbb{C}$, we prove that some power of $I$ admits a reduction formed by homogeneous polynomials with respect to the Newton filtration induced by $J$ if and only if the quotient of multiplicities $e(I) / e(J)$ attains a suitable upper bound expressed in terms of the Newton polyhedra of $I$ and $J$. We also explore other connections between mixed multiplicities, Newton filtrations and the integral closure of ideals.
\end{abstract}

\section{INTRODUCTION}

Let us denote by $\mathcal{O}_{n}$ the ring of complex analytic function germs $f:\left(\mathbb{C}^{n}, 0\right) \rightarrow \mathbb{C}$. Let $g:\left(\mathbb{C}^{n}, 0\right) \rightarrow\left(\mathbb{C}^{n}, 0\right)$ be a complex analytic map. We say that $g$ is finite when $g^{-1}(0)=\{0\}$; in this case, we refer to the number $e(g)=\operatorname{dim}_{\mathbb{C}} \mathcal{O}_{n} / I(g)$ as the multiplicity of $g$, where $I(g)$ denotes the ideal of $\mathcal{O}_{n}$ generated by the components of $g$ (see [1, §5], [8, §2] or [9, §2] for several characterizations of this number). More generally, if $I$ is any ideal of $\mathcal{O}_{n}$ of finite colength, then the multiplicity of $I$, in the sense of Hilbert-Samuel, is denoted by $e(I)$ (see [10, 12, 23]). We recall that, when $I$ admits a generating system formed by $n$ elements, then $e(I)=\operatorname{dim}_{\mathbb{C}} \mathcal{O}_{n} / I$. It is well-known that, if we fix a vector $w=\left(w_{1}, \ldots, w_{n}\right) \in \mathbb{Z}_{\geqslant 1}^{n}$ and $g$ is semi-weighted homogeneous with respect to $w$, then $e(g)$ can be expressed as

$$
e(g)=\frac{d_{1} \cdots d_{n}}{w_{1} \cdots w_{n}}
$$

where $d_{i}$ is the degree of $g_{i}$ with respect to $w$, for all $i=1, \ldots, n$ (see for instance [1, $\S 12.3$ ] or [8, §10.3]). This result was generalized in [7] by replacing the weighted homogeneous filtration induced by $w$ by the Newton filtration induced by a given Newton polyhedron of $\mathbb{R}_{\geqslant 0}^{n}$ (see Theorem 4.2). That is, let $\Gamma_{+} \subseteq \mathbb{R}_{\geqslant 0}^{n}$ be a Newton polyhedron such that $\Gamma_{+} \neq \mathbb{R}_{\geqslant 0}^{n}$ and $\Gamma_{+}$intersects each coordinate axis. Let $\Gamma$ be the union of all compact faces of $\Gamma_{+}$and let $\nu_{\Gamma}$ be the Newton filtration induced by $\Gamma_{+}$(see Section 4 for details). If $g:\left(\mathbb{C}^{n}, 0\right) \rightarrow\left(\mathbb{C}^{n}, 0\right)$ is any finite analytic map, then

$$
e(g) \geqslant \frac{d_{1} \cdots d_{n}}{M_{\Gamma}^{n}} n ! \mathrm{V}_{n}\left(\mathbb{R}_{\geqslant 0}^{n} \backslash \Gamma_{+}\right),
$$

2010 Mathematics Subject Classification. Primary 13H15; Secondary 13B22, 32S05.

Key words and phrases. Integral closure of ideals, mixed multiplicities of ideals, monomial ideals, Newton polyhedra.

The author was partially supported by DGICYT Grant MTM2015-64013-P. 
where $d_{i}=\nu_{\Gamma}\left(g_{i}\right)$, for all $i=1, \ldots, n, \mathrm{~V}_{n}$ denotes the $n$-dimensional volume and $M_{\Gamma}$ is the value of $\nu_{\Gamma}$ over the monomials whose exponent belongs to $\Gamma$. The maps $g:\left(\mathbb{C}^{n}, 0\right) \rightarrow\left(\mathbb{C}^{n}, 0\right)$ for which equality holds in (1) are called non-degenerate on $\Gamma_{+}$. This class of maps is characterized in [7, Theorem 3.3].

If $K$ is a monomial ideal of $\mathcal{O}_{n}$ of finite colength, then we recall that the multiplicity of $K$ is expressed as $e(K)=n ! \mathrm{V}_{n}\left(\mathbb{R}_{\geqslant 0}^{n} \backslash \Gamma_{+}(K)\right)$, where $\Gamma_{+}(K)$ denotes the Newton polyhedron of $K$ (see for instance [21, 22]). Therefore, relation (1) also shows a lower bound for the quotient $e(g) / e(J)$, where $J$ is the integrally closed monomial ideal such that $\Gamma_{+}=\Gamma_{+}(J)$. We also refer to non-degenerate maps on $\Gamma_{+}$as $J$-non-degenerate maps. We show that equality holds in (1) if and only if there exists some integers $a_{1}, \ldots, a_{n}, d \in \mathbb{Z}_{\geqslant 1}$ such that $\overline{\left\langle g_{1}^{a_{1}}, \ldots, g_{n}^{a_{n}}\right\rangle}=\overline{J^{d}}$, where the bar denotes integral closure.

Moreover, if $I$ is a monomial ideal of $\mathcal{O}_{n}$ of finite colength, then we use the respective Newton polyhedra of $I$ and $J$ to define an increasing sequence of positive rational numbers $a_{1, J}(I), \ldots, a_{n, J}(I)$ that leads to an upper bound for the quotient $e(I) / e(J)$, that is,

$$
\frac{e(I)}{e(J)} \leqslant \frac{a_{1, J}(I) \cdots a_{n, J}(I)}{M_{J}^{n}}
$$

where $M_{J}$ is a positive integer defined in terms of the Newton filtration of $J$ (see Section 4). We prove that equality holds in (2) if and only if there exists some $s \geqslant 1$ such that $\overline{I^{s}}=\overline{\left\langle g_{1}, \ldots, g_{n}\right\rangle}$, for some map $\left(g_{1}, \ldots, g_{n}\right):\left(\mathbb{C}^{n}, 0\right) \rightarrow\left(\mathbb{C}^{n}, 0\right)$ which is $J$-non-degenerate. This result appears in Theorem 5.5. The proof of this result is preceded by a characterization of the notion of $J$-non-degeneracy of $n$-tuples of monomial ideals (see Theorem 4.10 and Definition 4.3), which in turn depends on previous combinatorial results proven in Section 3. Let us remark that, by interchanging the roles of $I$ and $J$ in (2) we automatically obtain a lower bound for $e(I) / e(J)$ (see Corollary 5.7).

The motivation of our work in this article arises from our previous work [4, where we characterized when the integral closure of a given monomial ideal of $\mathcal{O}_{n}$ of finite colength is equal to the integral closure of the ideal generated by $n$ homogeneous polynomials. In turn, [4] was motivated by the results of Hickel in [11.

The article is organized as follows. In Section 2 we recall some definitions and results related with mixed multiplicities, joint reductions of families of ideals and Newton polyhedra that we will need in the article. Let $I_{1}, \ldots, I_{n}$ be $n$ monomial ideals of $\mathcal{O}_{n}$. Due to its importance in subsequent sections, we recall in Theorem 2.3 the result of Rees and Sally (see [15, Theorem 1.6] and [12, §17.3]) about the existence of joint reductions of $\left(I_{1}, \ldots, I_{n}\right)$, in the sense of Rees [14].

Section 3 is devoted to showing a combinatorial characterization of the finiteness of $\sigma\left(I_{1}, \ldots, I_{n}\right)$ (see Theorem 3.2), where $\sigma\left(I_{1}, \ldots, I_{n}\right)$ denotes what we call the Rees' mixed multiplicity of $I_{1}, \ldots, I_{n}$ (see (5)) and $I_{1}, \ldots, I_{n}$ are monomial ideals of $\mathcal{O}_{n}$. This result will be fundamental in the proofs of some results of Section 4 .

The objective of Section 4 is to show a combinatorial characterization of those pairs formed by an $n$-tuple $\left(I_{1}, \ldots, I_{n}\right)$ of monomial ideals of $\mathcal{O}_{n}$ such that $\sigma\left(I_{1}, \ldots, I_{n}\right)<\infty$ and 
a monomial ideal $J$ of $\mathcal{O}_{n}$ of finite colength for which $\left(I_{1}, \ldots, I_{n}\right)$ is $J$-non-degenerate (see Definition 4.3, which is a generalization of the notion of $J$-non-degenerate map. This is given in Theorem 4.10 .

In Section 5 we prove the existence of what we call central maps with respect to a given pair of monomial ideals of $\mathcal{O}_{n}$ of finite colength (see Theorem 5.3 and Corollary 5.4). The existence of central maps supports the proof of the upper bound mentioned in (2) and the characterization of the equality in (2) (see Theorem 5.5). We remark that in Corollary 5.7 (c) we show a characterization of the equality in (2) that is expressed only in terms of the respective Newton filtrations induced by $I$ and $J$.

\section{Preliminary CONCEPTS}

This section is devoted to recalling some definitions and fundamental facts that we will use along the paper.

\subsection{Mixed multiplicities and joint reductions}

Along this section we suppose that $(R, \mathbf{m})$ is a Noetherian local ring with infinite residue field $\mathbf{k}=R / \mathbf{m}$ and of dimension $n$. We recall some concepts and results from [2, 3, 5]. If $I$ is an ideal of $R$, then we denote by $\bar{I}$ the integral closure of $I$ (see [10, 12, 23]).

Let $I_{1}, \ldots, I_{n}$ be ideals of $R$ of finite colength. We denote by $e\left(I_{1}, \ldots, I_{n}\right)$ the mixed multiplicity of $I_{1}, \ldots, I_{n}$ defined by Teissier and Risler in [19, §2] (see also [12, Section 17.4] or [16, Section 2.5]). We recall briefly the definition of $e\left(I_{1}, \ldots, I_{n}\right)$. Let us consider the function $H: \mathbb{Z}_{\geqslant 0}^{n} \rightarrow \mathbb{Z}_{\geqslant 0}$ given by

$$
H\left(r_{1}, \ldots, r_{n}\right)=\ell\left(\frac{R}{I_{1}^{r_{1}} \cdots I_{n}^{r_{n}}}\right),
$$

for all $\left(r_{1}, \ldots, r_{n}\right) \in \mathbb{Z}_{\geqslant 0}^{n}$, where $\ell(M)$ denotes the length of a given $R$-module $M$. It is proven in [19, §2] that there exists a polynomial $P\left(x_{1}, \ldots, x_{n}\right) \in \mathbb{Q}\left[x_{1}, \ldots, x_{n}\right]$ of degree $n$ such that

$$
H\left(r_{1}, \ldots, r_{n}\right)=P\left(r_{1}, \ldots, r_{n}\right),
$$

for all sufficiently large $r_{1}, \ldots, r_{n} \in \mathbb{Z}_{\geqslant 0}$. Moreover, the coefficient of the monomial $x_{1} \cdots x_{n}$ in $P\left(x_{1}, \ldots, x_{n}\right)$ is a positive integer. This integer is called the mixed multiplicity of $I_{1}, \ldots, I_{n}$ and is denoted by $e\left(I_{1}, \ldots, I_{n}\right)$.

We remark that if $I_{1}, \ldots, I_{n}$ are all equal to a given ideal $I$ of finite colength of $R$, then $e\left(I_{1}, \ldots, I_{n}\right)=e(I)$, where $e(I)$ denotes the Samuel multiplicity of $I$. We refer to [12, $\S 17.4$ ] or [18] for fundamental results concerning mixed multiplicities of ideals.

Moreover Rees showed in [14] that the mixed multiplicity $e\left(I_{1}, \ldots, I_{n}\right)$ can be computed in terms of Samuel multiplicities via the following formula:

$$
e\left(I_{1}, \ldots, I_{n}\right)=\frac{1}{n !} \sum_{\substack{\mathrm{J} \subseteq\{1, \ldots, n\} \\ \mathrm{J} \neq \emptyset}}(-1)^{n-|\mathrm{J}|} e\left(\prod_{j \in \mathrm{J}} I_{j}\right),
$$

where we denote by $|X|$ the cardinal of a given finite set $X$. 
Given two ideals $I$ and $J$ of $R$ of finite colength and an integer $i \in\{1, \ldots, n\}$, we define

$$
e_{i}(I, J)=e(I, \ldots, I, J, \ldots, J),
$$

where $I$ is repeated $i$ times and $J$ is repeated $n-i$ times.

Let $I_{1}, \ldots, I_{n}$ be proper ideals of $R$ (not necessarily of finite colength). In [2] we studied the following number:

$$
\sigma\left(I_{1}, \ldots, I_{n}\right)=\sup _{r \in \mathbb{Z} \geqslant 0} e\left(I_{1}+\mathbf{m}^{r}, \ldots, I_{n}+\mathbf{m}^{r}\right) .
$$

When the set of integers $\left\{e\left(I_{1}+\mathbf{m}^{r}, \ldots, I_{n}+\mathbf{m}^{r}\right): r \in \mathbb{Z}_{\geqslant 0}\right\}$ is bounded, then we refer to $\sigma\left(I_{1}, \ldots, I_{n}\right)$ as the Rees' mixed multiplicity of $I_{1}, \ldots, I_{n}$. Obviously, if $I_{i}$ has finite colength, for all $i=1, \ldots, n$, then $\sigma\left(I_{1}, \ldots, I_{n}\right)=e\left(I_{1}, \ldots, I_{n}\right)$.

In Proposition 2.2 we recall a result from [2] that interprets $\sigma\left(I_{1}, \ldots, I_{n}\right)$ as a multiplicity in the usual sense. First we need to introduce a preliminary concept.

Definition 2.1. Let $I_{1}, \ldots, I_{r}$ be proper ideals of $R$. Let $a_{i 1}, \ldots, a_{i s_{i}}$ be a minimal generating system of $I_{i}$, where $s_{i} \in \mathbb{Z}_{\geqslant 1}$, for all $i=1, \ldots, r$. Let $s=s_{1}+\cdots+s_{r}$. We say that a property holds for sufficiently general elements $\left(g_{1}, \ldots, g_{r}\right) \in I_{1} \oplus \cdots \oplus I_{r}$ if there exists a non-empty Zariski-open set $U$ in $\mathbf{k}^{s}$ verifying that if

(a) $g_{i}=\sum_{j} u_{i j} a_{i j}$, where $u_{i j} \in R$, for all $j=1, \ldots, s_{i}, i=1, \ldots, r$, and

(b) the image of $\left(u_{11}, \ldots, u_{1 s_{1}}, \ldots, u_{r 1}, \ldots, u_{r s_{r}}\right)$ in $\mathbf{k}^{s}$ belongs to $U$,

then the said property holds for $\left(g_{1}, \ldots, g_{r}\right)$.

Proposition 2.2. [2, 2.9] Let $I_{1}, \ldots, I_{n}$ be proper ideals of $R$. Then $\sigma\left(I_{1}, \ldots, I_{n}\right)<\infty$ if and only if there exist elements $g_{i} \in I_{i}$, for $i=1, \ldots, n$, such that $\left\langle g_{1}, \ldots, g_{n}\right\rangle$ has finite colength. If $\sigma\left(I_{1}, \ldots, I_{n}\right)<\infty$, then $\sigma\left(I_{1}, \ldots, I_{n}\right)=e\left(g_{1}, \ldots, g_{n}\right)$ for sufficiently general elements $\left(g_{1}, \ldots, g_{n}\right) \in I_{1} \oplus \cdots \oplus I_{n}$.

Let $I$ and $J$ be ideals of $R$ such that $J \subseteq I$. We recall that an ideal $J$ is called a reduction of $I$ if there exists some $r \in \mathbb{Z}_{\geqslant 0}$ such that $I^{r+1}=J I^{r}$. It is well known that $J$ is a reduction of $I$ if and only if $\bar{I}=\bar{J}$ (see for instance [12, Corollary 1.2.5]). In turn, if we assume that the ideals $I$ and $J$ have finite colength, $J \subseteq I$ and $R$ is quasi-unmixed, then the celebrated Rees' multiplicity theorem says that the equality $\bar{I}=\bar{J}$ holds if and only if $e(I)=e(J)$ (see [10, p. 147] or [12, p. 222]).

Let $I_{1}, \ldots, I_{n}$ be ideals of $R$. Let $g_{1}, \ldots, g_{n} \in R$ such that $g_{i} \in I_{i}$, for all $i=1, \ldots, n$. Then $\left(g_{1}, \ldots, g_{n}\right)$ is called a joint reduction of $\left(I_{1}, \ldots, I_{n}\right)$ when $g_{1} I_{2} \cdots I_{n}+\cdots+g_{n} I_{1} \cdots I_{n-1}$ is a reduction of $I_{1} \cdots I_{n}$. By the relation between reductions and integral closure mentioned before, this condition is equivalent to saying that

$$
\overline{g_{1} I_{2} \cdots I_{n}+\cdots+g_{n} I_{1} \cdots I_{n-1}}=\overline{I_{1} \cdots I_{n}} .
$$

Let us fix a family $I_{1}, \ldots, I_{p}$ of proper ideals of $R$. We recall that $\operatorname{dim}(R)=n$. In [14, Theorem 1.3], D. Rees showed that there exists a family of elements $\left\{x_{i j}: i=1, \ldots, p, j=\right.$ $1, \ldots n\} \subseteq R$ such that $x_{i 1}, \ldots, x_{i n} \in I_{i}$, for all $i=1, \ldots, p$, and if $y_{j}=x_{1 j} \cdots x_{p j}$, for 
all $j=1, \ldots, n$, then the ideal $\left\langle y_{1}, \ldots, y_{n}\right\rangle$ is a reduction of the product ideal $I_{1} \cdots I_{p}$. We remark that $p$ is not assumed to be equal to $n$ in this result. Any set of elements $x_{i j}$ satisfying the above properties is called a complete reduction of $\left(I_{1}, \ldots, I_{p}\right)$ (see [14, p. 402] or [12, Definition 17.1.3]).

Now let us suppose that $p=n$. In [14, Theorem 1.4] D. Rees easily proved that, if $\left\{x_{i j}: i, j=1, \ldots, n\right\}$ is a complete reduction of $\left(I_{1}, \ldots, I_{n}\right)$, then $x_{1 \sigma(1)}, \ldots, x_{n \sigma(n)}$ is a joint reduction of $\left(I_{1}, \ldots, I_{n}\right)$, for any permutation $i \mapsto \sigma(i)$ of $\{1, \ldots, n\}$. In [15, Theorem 1.6] Rees and Sally proved that joint reductions of sets of $n$ ideals exist, we next recall this result (see also [12, §17.3]).

Theorem 2.3. Let $I_{1}, \ldots, I_{n}$ be ideals of $R$. Then $\left(g_{1}, \ldots, g_{n}\right)$ is a joint reduction of $\left(I_{1}, \ldots, I_{n}\right)$, for sufficiently general elements $\left(g_{1}, \ldots, g_{n}\right) \in I_{1} \oplus \cdots \oplus I_{n}$.

\subsection{Newton polyhedra of ideals and non-degeneracy conditions}

Let us fix a coordinate system $x_{1}, \ldots, x_{n}$ in $\mathbb{C}^{n}$. If $k=\left(k_{1}, \ldots, k_{n}\right) \in \mathbb{Z}_{\geqslant 0}^{n}$, then we denote the monomial $x_{1}^{k_{1}} \cdots x_{n}^{k_{n}}$ by $x^{k}$. Given a proper ideal $J$ of $\mathcal{O}_{n}$, we will say that $J$ is monomial when $J$ admits a generating system formed by monomials.

If $h \in \mathcal{O}_{n}$ and $h=\sum_{k} a_{k} x^{k}$ is the Taylor expansion of $h$ around the origin, then the support of $h$, denoted by $\operatorname{supp}(h)$ is defined as the set $\left\{k \in \mathbb{Z}_{\geqslant 0}^{n}: a_{k} \neq 0\right\}$. Given a subset $\Delta \subseteq \mathbb{R}_{\geqslant 0}^{n}$, we denote by $h_{\Delta}$ the sum of those terms $a_{k} x^{k}$ such that $k \in \Delta \cap \operatorname{supp}(h)$. If $\Delta \cap \operatorname{supp}(h)=\emptyset$, then we set $h_{\Delta}=0$. If $I$ is an ideal of $\mathcal{O}_{n}$, then the support of $I$ is defined as $\operatorname{supp}(I)=\cup_{g \in I} \operatorname{supp}(g)$.

If $A \subseteq \mathbb{Z}_{\geqslant 0}^{n}, A \neq \emptyset$, then we define the Newton polyhedron determined by $A$, denoted by $\Gamma_{+}(A)$, as the convex hull in $\mathbb{R}^{n}$ of the set $\left\{k+v: k \in A, v \in \mathbb{R}_{\geqslant 0}^{n}\right\}$. A subset $\Gamma_{+} \subseteq \mathbb{R}_{\geqslant 0}^{n}$ is called a Newton polyhedron when $\Gamma_{+}=\Gamma_{+}(A)$, for some $A \subseteq \mathbb{Z}_{\geqslant 0}^{n}$.

Let us fix a Newton polyhedron $\Gamma_{+} \subseteq \mathbb{R}_{\geqslant 0}^{n}$. If $v \in \mathbb{R}_{\geqslant 0}^{n}$, then we define

$$
\begin{aligned}
\ell\left(v, \Gamma_{+}\right) & =\min \left\{\langle v, k\rangle: k \in \Gamma_{+}\right\} \\
\Delta\left(v, \Gamma_{+}\right) & =\left\{k \in \Gamma_{+}:\langle v, k\rangle=\ell\left(v, \Gamma_{+}\right)\right\},
\end{aligned}
$$

where $\langle$,$\rangle stands for the standard scalar product in \mathbb{R}^{n}$. A face of $\Gamma_{+}$is any set $\Delta$ of the form $\Delta=\Delta\left(v, \Gamma_{+}\right)$, for some $v \in \mathbb{R}_{\geqslant 0}^{n} \backslash\{0\}$; in this case we say that $\Delta$ is supported by $v$. Given a face $\Delta$ of $\Gamma_{+}$, we observe that $\Delta$ is compact if and only if it is supported by a vector $v \in \mathbb{R}_{>0}^{n}$. The dimension of $\Delta$ is defined as the minimum of the dimensions of the affine subspaces of $\mathbb{R}^{n}$ containing $\Delta$. We denote by $C(\Delta)$ the cone formed by all half-lines emanating from the origin and passing through some point of $\Delta$. Let us denote by $\mathcal{R}_{\Delta}$ the subring of $\mathcal{O}_{n}$ formed by the functions $h \in \mathcal{O}_{n}$ such that $\operatorname{supp}(h) \subseteq C(\Delta)$.

The faces of dimension 0 and the faces of dimension $n-1$ of $\Gamma_{+}$are known, respectively, as the vertices and the facets of $\Gamma_{+}$. We denote by $\mathbf{v}\left(\Gamma_{+}\right)$the set of vertices of $\Gamma_{+}$. The union of all compact faces of $\Gamma_{+}$will be denoted by $\Gamma$. We will refer to $\Gamma$ as the Newton boundary of $\Gamma_{+}$. We remark that $\Gamma_{+}$is univocally determined by the set $\Gamma$, since $\Gamma_{+}=\Gamma+\mathbb{R}_{\geqslant 0}^{n}$. We denote by $\Gamma_{-}$the union of all segments joining the origin and some point of $\Gamma$. 
If $h \in \mathcal{O}_{n}$, then the Newton polyhedron of $h$ is defined as $\Gamma_{+}(h)=\Gamma_{+}(\operatorname{supp}(h))$. Moreover, if $I$ is an ideal of $\mathcal{O}_{n}$, then the Newton polyhedron of $I$ is defined as $\Gamma_{+}(I)=\Gamma_{+}(\operatorname{supp}(I))$. We recall that $\Gamma_{+}(I)=\Gamma_{+}(\bar{I})$ (see for instance [7, Lemma 2.3]).

If $\left\{g_{1}, \ldots, g_{r}\right\}$ is a generating set of $I$, then it is straightforward to see that $\Gamma_{+}(I)$ equals the convex hull of $\Gamma_{+}\left(g_{1}\right) \cup \cdots \cup \Gamma_{+}\left(g_{r}\right)$. We denote the Newton boundary of $\Gamma_{+}(I)$ by $\Gamma(I)$ and the union of all segments joining the origin with some point of $\Gamma(I)$ by $\Gamma_{-}(I)$.

Let $I$ be a proper ideal of $\mathcal{O}_{n}$ and let $g_{1}, \ldots, g_{s}$ be a generating system of $I$. We recall that $I$ is called Newton non-degenerate (see [2, 17, 17]) when

$$
\left\{x \in \mathbb{C}^{n}:\left(g_{1}\right)_{\Delta}(x)=\cdots=\left(g_{s}\right)_{\Delta}(x)=0\right\} \subseteq\left\{x \in \mathbb{C}^{n}: x_{1} \cdots x_{n}=0\right\},
$$

as set germs at $0 \in \mathbb{C}^{n}$, for each compact face $\Delta$ of $\Gamma_{+}(I)$. It was proven by Saia [17] that an ideal $I$ of $\mathcal{O}_{n}$ is Newton non-degenerate if and only if the integral closure of $I$ is equal to the ideal generated by those monomials $x^{k}$ such that $k \in \Gamma_{+}(I)$ (see also [7, Corollary 2.6] or [2, Proposition 3.6]).

As a consequence of [2, Proposition 3.6] we have that, if $I$ is a monomial ideal of $\mathcal{O}_{n}$ and $J \subseteq I$, then $J$ is a reduction of $I$ if and only if $J$ is Newton non-degenerate and $\Gamma_{+}(I)=\Gamma_{+}(J)$.

We remark that, if $f \in \mathcal{O}_{n}$, then the condition of Newton non-degeneracy of the ideal $\left\langle x_{1} \frac{\partial f}{\partial x_{1}}, \ldots, x_{n} \frac{\partial f}{\partial x_{n}}\right\rangle$ allows to obtain a lot of information about the topology of $f$ by means of $\Gamma_{+}(f)$ (see [13] and [24]).

\section{The ReEs' miXed multiplicity of A FAmily of MONOMial ideAls}

Given a non-empty subset $\mathrm{L} \subseteq\{1, \ldots, n\}$, if $\mathbb{K}=\mathbb{R}$ or $\mathbb{C}$, then we define $\mathbb{K}_{\mathrm{L}}^{n}=\{x \in$ $\mathbb{K}^{n}: x_{i}=0$, for all $\left.i \notin \mathrm{L}\right\}$. Let $r=|\mathrm{L}|$ and let us write $\mathrm{L}=\left\{j_{1}, \ldots, j_{r}\right\}$, for some integers $1 \leqslant j_{1}<\cdots<j_{r} \leqslant n$. If $S \subseteq \mathbb{K}^{n}$, then we denote by $S^{\mathrm{L}}$ the intersection $S \cap \mathbb{K}_{\mathrm{L}}^{n}$. Let us define

$$
\begin{aligned}
\mathbf{H} & =\left\{x \in \mathbb{C}^{n}: x_{1} \cdots x_{n}=0\right\} \\
\mathbf{H}_{\mathrm{L}} & =\left\{\left(x_{j_{1}}, \ldots, x_{j_{r}}\right) \in \mathbb{C}^{r}: x_{j_{1}} \cdots x_{j_{r}}=0\right\} .
\end{aligned}
$$

Hence, if $|\mathrm{L}|=1$, then $\mathbf{H}_{\mathrm{L}}=\{0\} \subseteq \mathbb{C}$.

If we fix a non-empty subset $\mathrm{L} \subseteq\{1, \ldots, n\}$, then $h^{\mathrm{L}}$ will denote the sum of all terms $a_{k} x^{k}$, such that $k \in \operatorname{supp}(h)^{\mathrm{L}}$. Then $\operatorname{supp}\left(h^{\mathrm{L}}\right)=\operatorname{supp}(h)^{\mathrm{L}}$. Let $\mathcal{O}_{n, \mathrm{~L}}$ denote the subring of $\mathcal{O}_{n}$ formed by all elements $h \in \mathcal{O}_{n}$ with $\operatorname{supp}(h) \subseteq \mathbb{R}_{\mathrm{L}}^{n}$. Let us remark that the map $\pi_{\mathrm{L}}: \mathcal{O}_{n} \rightarrow \mathcal{O}_{n, \mathrm{~L}}$ given by $\pi_{\mathrm{L}}(h)=h^{\mathrm{L}}$, for all $h \in \mathcal{O}_{n}$, is a ring morphism. In order to simplify the notation, if $I$ is an ideal of $\mathcal{O}_{n}$, then we also denote the ideal $\pi_{\mathrm{L}}(I)$ by $I^{\mathrm{L}}$.

If $I=\left\langle g_{1}, \ldots, g_{r}\right\rangle$ is an ideal of $\mathcal{O}_{n}$, then we denote by $\mathbf{V}(I)$, or by $\mathbf{V}\left(g_{1}, \ldots, g_{r}\right)$, the zero set germ of $I$ at 0 . Let $\mathbf{m}_{n}$ denote the maximal ideal of $\mathcal{O}_{n}$.

Proposition 3.1. Let $I$ be a proper ideal of $\mathcal{O}_{n}$. Then $I$ has finite colength if and only if $\mathrm{V}\left(I^{\mathrm{L}}\right) \subseteq \mathbf{H}_{\mathrm{L}}$, for all $\mathrm{L} \subseteq\{1, \ldots, n\}, \mathrm{L} \neq \emptyset$. 
Proof. Let us prove first the only if part. Let us fix a subset $\mathrm{L} \subseteq\{1, \ldots, n\}, \mathrm{L} \neq \emptyset$. Since $I$ has finite colength, there exists some integer $r \geqslant 1$ such that $\mathbf{m}^{r} \subseteq I$. If we apply $\pi_{\mathrm{L}}$ to both sides of this inclusion, we conclude that the ideal $I^{\mathrm{L}}$ has also finite colength in $\mathcal{O}_{n, \mathrm{~L}}$. In particular, $\mathbf{V}\left(I^{\mathrm{L}}\right)=\{0\} \subseteq \mathbf{H}_{\mathrm{L}}$.

Let us prove the if part. Let $g_{1}, \ldots, g_{r}$ be any generating system of $I$ and let $\mathrm{L} \subseteq\{1, \ldots, n\}$, $\mathrm{L} \neq \emptyset$. The condition $\mathbf{V}\left(I^{\mathrm{L}}\right) \subseteq \mathbf{H}_{\mathrm{L}}$ implies that $I^{\mathrm{L}} \neq 0$; otherwise $\mathbf{V}\left(I^{\mathrm{L}}\right)=\mathbb{C}_{\mathrm{L}}^{n}$. Therefore $\left\{i: g_{i}^{\mathrm{L}} \neq 0\right\} \neq \emptyset$. Then we have that

$$
\mathbf{V}(I) \cap \mathbb{C}_{\mathrm{L}}^{n}=\mathbf{V}\left(g_{1}, \ldots, g_{r}\right) \cap \mathbb{C}_{\mathrm{L}}^{n}=\mathbf{V}\left(g_{1}^{\mathrm{L}}, \ldots, g_{r}^{\mathrm{L}}\right)=\mathbf{V}\left(I^{\mathrm{L}}\right) .
$$

By the same reason, we have

$$
\mathbf{V}\left(I^{\mathrm{L}^{\prime}}\right) \cap \mathbb{C}_{\mathrm{L}}^{n}=\mathbf{V}\left(I^{\mathrm{L}}\right)
$$

for all $\mathrm{L}^{\prime} \subseteq\{1, \ldots, n\}$ containing L.

Let $\mathrm{L} \subseteq\{1, \ldots, n\}$ such that $|\mathrm{L}|=1$. By hypothesis we have $\mathrm{V}\left(I^{\mathrm{L}}\right) \subseteq \mathbf{H}_{\mathrm{L}}=\{0\} \subseteq \mathbb{C}$. Since $I \subseteq \mathbf{m}_{n}$, we have $I^{\mathrm{L}} \subseteq \mathbf{m}_{n}^{\mathrm{L}}$ and then $\{0\} \subseteq \mathbf{V}\left(I^{\mathrm{L}}\right)$. Thus $\mathbf{V}\left(I^{\mathrm{L}}\right)=\{0\}$.

Let $j \in\{1, \ldots, n-1\}$ and let us suppose that $\mathbf{V}\left(I^{\mathrm{L}}\right)=\{0\}$, for all $\mathrm{L} \subseteq\{1, \ldots, n\}$ such that $|\mathrm{L}| \leqslant j$. Let us fix a subset $\mathrm{L}^{\prime} \subseteq\{1, \ldots, n\}$ such that $\left|\mathrm{L}^{\prime}\right|=j+1$. The condition $\mathbf{V}\left(I^{\mathrm{L}^{\prime}}\right) \subseteq \mathbf{H}_{\mathrm{L}^{\prime}}$ implies that

$$
\mathbf{V}\left(I^{\mathrm{L}^{\prime}}\right)=\bigcup_{\substack{\mathrm{L} \subseteq\{1, \ldots, n\} \\|\mathrm{L}|=j, \mathrm{~L} \subseteq \mathrm{L}^{\prime}}}\left(\mathbf{V}(I) \cap \mathbb{C}_{\mathrm{L}}^{n}\right)=\bigcup_{\substack{\mathrm{L} \subseteq\{1, \ldots, n\} \\|\mathrm{L}|=j, \mathrm{~L} \subseteq \mathrm{L}^{\prime}}} \mathbf{V}\left(I^{\mathrm{L}}\right)
$$

where the first equality comes from (7). Then $\mathbf{V}\left(I^{L^{\prime}}\right)=\{0\}$. By finite induction on $|\mathrm{L}|$ we deduce that $\mathbf{V}(I)=\mathbf{V}\left(I^{\{1, \ldots, n\}}\right)=\{0\}$.

Theorem 3.2. Let $I_{1}, \ldots, I_{n}$ be monomial ideals of $\mathcal{O}_{n}$. Then the following conditions are equivalent.

(a) $\sigma\left(I_{1}, \ldots, I_{n}\right)<\infty$.

(b) For each $\mathrm{L} \subseteq\{1, \ldots, n\}, \mathrm{L} \neq \emptyset$, we have $\left|\left\{i: I_{i}^{\mathrm{L}} \neq 0\right\}\right| \geqslant|\mathrm{L}|$.

Proof. Let us prove $(\mathrm{a}) \Rightarrow(\mathrm{b})$. If $\sigma\left(I_{1}, \ldots, I_{n}\right)<\infty$, then there exist some $\left(g_{1}, \ldots, g_{n}\right) \in$ $I_{1} \oplus \cdots \oplus I_{n}$ such that, if $I$ denotes the ideal generated by $g_{1}, \ldots, g_{n}$, then $I$ has finite colength in $\mathcal{O}_{n}$ and $e(I)=\sigma\left(I_{1}, \ldots, I_{n}\right)$, by Proposition 2.2. In particular, there exists some $r \in \mathbb{Z}_{\geqslant 1}$ such that $\mathbf{m}_{n}^{r} \subseteq I$. Given a subset $\mathrm{L} \subseteq\{1, \ldots, n\}, \mathrm{L} \neq \emptyset$, if we apply $\pi_{\mathrm{L}}$ to both sides of the inclusion $\mathbf{m}_{n}^{r} \subseteq I$, then we obtain that the ideal $I^{\mathrm{L}}$ has also finite colength. This implies that the set $\left\{g_{1}^{\mathrm{L}}, \ldots, g_{n}^{\mathrm{L}}\right\}$ contains at least $|\mathrm{L}|$ non-zero elements, since $\operatorname{dim} \mathcal{O}_{n, \mathrm{~L}}=|\mathrm{L}|$. Then, as $\operatorname{supp}\left(g_{i}^{\mathrm{L}}\right) \subseteq I_{i}^{\mathrm{L}}$, for all $i=1, \ldots, n$, condition (b) holds.

Let us prove $(\mathrm{b}) \Rightarrow(\mathrm{a})$. By Proposition 2.2, it suffices to see that there exists an element $\left(g_{1}, \ldots, g_{n}\right) \in I_{1} \oplus \cdots \oplus I_{n}$ such that the ideal generated by $g_{1}, \ldots, g_{n}$ in $\mathcal{O}_{n}$ has finite colength. 
Let us fix a subset $\mathrm{L} \subseteq\{1, \ldots, n\}, \mathrm{L} \neq \emptyset$. By hypothesis we can choose a set of indexes $B_{\mathrm{L}} \subseteq\{1, \ldots, n\}$ such that $\left|B_{\mathrm{L}}\right|=|\mathrm{L}|$ and $I_{j}^{\mathrm{L}} \neq 0$, for all $j \in B_{\mathrm{L}}$. Let us write $B_{\mathrm{L}}$ as $B_{\mathrm{L}}=\left\{j_{\mathrm{L}, 1}, \ldots, j_{\mathrm{L},|\mathrm{L}|}\right\} \subseteq\{1, \ldots, n\}$, for some integers $1 \leqslant j_{\mathrm{L}, 1}<\cdots<j_{\mathrm{L},|\mathrm{L}|} \leqslant n$.

Let $G_{i}$ be a fixed minimal generating system of $I_{i}$ formed by monomials, for all $i=$ $1, \ldots, n$, and let $\mathbf{G}=\left(G_{1}, \ldots, G_{n}\right)$. Let us denote by $G_{i}^{\mathrm{L}}$ be the set of monomials of $G_{i}$ whose support belongs to $\mathbb{R}_{\mathrm{L}}^{n}$. Hence $G_{i}^{\mathrm{L}}$ is a generating system of $I_{i}^{\mathrm{L}}$, for all $i=1, \ldots, n$. Let $\mathbf{G}^{\mathrm{L}}=\left(G_{j_{\mathrm{L}, 1}}^{\mathrm{L}}, \ldots, G_{j_{\mathrm{L},|\mathrm{L}|}}^{\mathrm{L}}\right)$. Let us identify the set of $\mathrm{G}^{\mathrm{L}}$-maps with $\mathbb{C}^{N_{\mathrm{L}}}$, where $N_{\mathrm{L}}=$ $\left|G_{j_{\mathrm{L}, 1}}^{\mathrm{L}}\right|+\cdots+\left|G_{j_{\mathrm{L}, \mathrm{L} \mid} \mid}^{\mathrm{L}}\right|$.

Since $\operatorname{dim} \mathcal{O}_{n, \mathrm{~L}}=|\mathrm{L}|$, we can apply Theorem 2.3 to the $|\mathrm{L}|$-tuple of ideals $\left(I_{j_{\mathrm{L}, 1}}^{\mathrm{L}}, \ldots, I_{j_{\mathrm{L}, \mathrm{L}} \mid}^{\mathrm{L}}\right)$. Thus, there exist a Zariski-open subset $U_{\mathrm{L}} \subseteq \mathbb{C}^{N_{\mathrm{L}}}$, such that any $\mathrm{G}^{\mathrm{L}}$-map with coefficients in $U_{\mathrm{L}}$ is a joint reduction of $\left(I_{j_{\mathrm{L}, 1}}^{\mathrm{L}}, \ldots, I_{j_{\mathrm{L},|\mathrm{L}|}}^{\mathrm{L}}\right)$. Hence there exists a G-map $\left(g_{1}, \ldots, g_{n}\right) \in$ $I_{1} \oplus \cdots \oplus I_{n}$ such that the set of coefficients of $\left(g_{j_{\mathrm{L}, 1}}^{\mathrm{L}}, \ldots, g_{j_{\mathrm{L},|\mathrm{L}|}}^{\mathrm{L}}\right)$ belongs to $U_{\mathrm{L}}$, for all nonempty $\mathrm{L} \subseteq\{1, \ldots, n\}$.

Let us fix again a subset $\mathrm{L} \subseteq\{1, \ldots, n\}, \mathrm{L} \neq \emptyset$. Since $\left(g_{j_{\mathrm{L}, 1}^{\mathrm{L}}}^{\mathrm{L}}, \ldots, g_{j_{\mathrm{L},|\mathrm{L}|}^{\mathrm{L}}}^{\mathrm{L}}\right)$ is a joint reduction of $\left(I_{j_{\mathrm{L}, 1}}^{\mathrm{L}}, \ldots, I_{j_{\mathrm{L},|\mathrm{L}|}}^{\mathrm{L}}\right)$, by $(6)$, we obtain that

$$
\overline{g_{j_{\mathrm{L}, 1}}^{\mathrm{L}} I_{j_{\mathrm{L}, 2}}^{\mathrm{L}} \cdots I_{j_{\mathrm{L},|\mathrm{L}|}^{\mathrm{L}}}^{\mathrm{L}}+\cdots+g_{j_{\mathrm{L},|\mathrm{L}|}^{\mathrm{L}}}^{\mathrm{L}} I_{j_{\mathrm{L}, 1}}^{\mathrm{L}} \cdots I_{j_{\mathrm{L},|\mathrm{L}|-1}^{\mathrm{L}}}^{\mathrm{L}}}=\overline{I_{j_{\mathrm{L}, 1}}^{\mathrm{L}} \cdots I_{j_{\mathrm{L}, \mathrm{L} \mid}}^{\mathrm{L}}} .
$$

Hence $\overline{I_{j_{\mathrm{L}, 1}}^{\mathrm{L}} \cdots I_{j_{\mathrm{L},|\mathrm{L}|}}^{\mathrm{L}}} \subseteq \overline{\left\langle g_{j_{\mathrm{L}, 1}}^{\mathrm{L}}, \ldots, g_{j_{\mathrm{L},|\mathrm{L}|}}^{\mathrm{L}}\right\rangle}$, which in turn implies that

$$
\mathbf{V}\left(g_{1}^{\mathrm{L}}, \ldots, g_{n}^{\mathrm{L}}\right) \subseteq \mathbf{V}\left(g_{j_{\mathrm{L}, 1}^{\mathrm{L}}}^{\mathrm{L}}, \ldots, g_{j_{\mathrm{L},|\mathrm{L}|}}^{\mathrm{L}}\right) \subseteq \mathbf{V}\left(I_{j_{\mathrm{L}, 1}}^{\mathrm{L}} \cdots I_{j_{\mathrm{L},|\mathrm{L}|}^{\mathrm{L}}}^{\mathrm{L}}\right) \subseteq \mathbf{H}_{\mathrm{L}}
$$

where the last inclusion follows from the fact that $I_{1}, \ldots, I_{n}$ are monomial ideals. Then we have deduced that $\mathbf{V}\left(g_{1}^{\mathrm{L}}, \ldots, g_{n}^{\mathrm{L}}\right) \subseteq \mathbf{H}_{\mathrm{L}}$, for all non-empty subsets $\mathrm{L} \subseteq\{1, \ldots, n\}$. In particular, the ideal $\left\langle g_{1}, \ldots, g_{n}\right\rangle$ has finite colength in $\mathcal{O}_{n}$, by Proposition 3.1, and the result follows.

\section{Characterization of $J$-NON-Degeneracy of Sequences of iDEals}

This section is devoted to characterize those sequences of ideals whose Rees' mixed multiplicity attains a lower bound formulated in terms of a fixed Newton filtration.

\subsection{The Newton filtration and the computation of multiplicities}

Let $\Gamma_{+} \subseteq \mathbb{R}^{n}$ be a Newton polyhedron. We say that $\Gamma_{+}$is convenient when $\Gamma_{+} \neq \mathbb{R}_{\geqslant 0}^{n}$ and $\Gamma_{+}$intersects each coordinate axis. If $\Gamma_{+}$is convenient, then $\Gamma_{-}$is equal to the closure of $\mathbb{R}_{\geqslant 0}^{n} \backslash \Gamma_{+}$(in the usual Euclidean sense). We observe that, if $I$ is an ideal of $\mathcal{O}_{n}$ of finite colength, then $\Gamma_{+}(I)$ is convenient.

Let $v \in \mathbb{Z}_{\geqslant 0}^{n}, v \neq 0$, we say that $v$ is primitive when the non-zero coordinates of $v$ are mutually prime integer numbers. Then any facet of $\Gamma_{+}$is supported by a unique primitive vector of $\mathbb{Z}_{\geqslant 0}^{n}$. Let us denote by $\mathcal{F}\left(\Gamma_{+}\right)$the set of primitive vectors of $\mathbb{Z}_{\geqslant 0}^{n}$ supporting some facet of $\Gamma_{+}$and let $\mathcal{F}_{c}\left(\Gamma_{+}\right)=\mathcal{F}\left(\Gamma_{+}\right) \cap \mathbb{Z}_{\geqslant 1}^{n}$. Let us remark that, if $\Gamma_{+}$is convenient, then $\mathcal{F}\left(\Gamma_{+}\right)=\mathcal{F}_{c}\left(\Gamma_{+}\right) \cup\left\{e_{1}, \ldots, e_{n}\right\}$, where $e_{1}, \ldots, e_{n}$ is the canonical basis of $\mathbb{R}^{n}$.

Let us fix a convenient Newton polyhedron $\Gamma_{+} \subseteq \mathbb{R}_{\geqslant 0}^{n}$. Let us write $\mathcal{F}_{c}\left(\Gamma_{+}\right)=\left\{v^{1}, \ldots, v^{r}\right\}$. Then $\ell\left(v^{i}, \Gamma_{+}\right)>0$, for all $i=1, \ldots, r$. We denote by $M_{\Gamma}$ the least common multiple of the 
set of integers $\left\{\ell\left(v^{1}, \Gamma_{+}\right), \ldots, \ell\left(v^{r}, \Gamma_{+}\right)\right\}$. We define the filtrating map associated to $\Gamma_{+}$as the map $\phi_{\Gamma}: \mathbb{R}_{\geqslant 0}^{n} \rightarrow \mathbb{R}_{\geqslant 0}$ given by

$$
\phi_{\Gamma}(k)=\min \left\{\frac{M_{\Gamma}}{\ell\left(v^{i}, \Gamma_{+}\right)}\left\langle k, v^{i}\right\rangle: i=1, \ldots, r\right\}, \quad \text { for all } k \in \mathbb{R}_{\geqslant 0}^{n} .
$$

We observe that $\phi_{\Gamma}\left(\mathbb{Z}_{\geqslant 0}^{n}\right) \subseteq \mathbb{Z}_{\geqslant 0}^{n}, \phi_{\Gamma}(k)=M_{\Gamma}$, for all $k \in \Gamma$, and the map $\phi_{\Gamma}$ is linear on each cone $C(\Delta)$, where $\Delta$ is any compact face of $\Gamma_{+}$. As mentioned in [13, p. 10], given $a, b \in \mathbb{Z}_{\geqslant 0}^{n}$, it is easy to prove that $\phi_{\Gamma}(a+b) \geqslant \phi_{\Gamma}(a)+\phi_{\Gamma}(b)$. Moreover, it is a straightforward exercise to see that equality holds if and only if there exists some compact face $\Delta$ of $\Gamma_{+}$such that $a, b \in C(\Delta)$.

Let us define the map $\nu_{\Gamma}: \mathcal{O}_{n} \rightarrow \mathbb{R}_{\geqslant 0} \cup\{+\infty\}$ by $\nu_{\Gamma}(h)=\min \left\{\phi_{\Gamma}(k): k \in \operatorname{supp}(h)\right\}$, for all $h \in \mathcal{O}_{n}, h \neq 0$; we set $\nu_{\Gamma}(0)=+\infty$. We refer to $\nu_{\Gamma}$ as the Newton filtration induced by $\Gamma_{+}$(see also [7, 13]).

By abuse of notation, if $A \subseteq \mathbb{R}_{\geqslant 0}^{n}$ denotes a non-empty closed set, then we define $\nu_{\Gamma}(A)=$ $\min \left\{\phi_{\Gamma}(k): k \in A\right\}$. Hence $\nu_{\Gamma}(h)=\nu_{\Gamma}(\operatorname{supp}(h))$, for all $h \in \mathcal{O}_{n}, h \neq 0$. If $I$ is a non-zero ideal of $\mathcal{O}_{n}$, then we also define $\nu_{\Gamma}(I)=\nu_{\Gamma}(\operatorname{supp}(I))$. Given a proper ideal $J$ of $\mathcal{O}_{n}$ of finite colength, then we denote by $\phi_{J}$ the filtrating map associated to $\Gamma_{+}(J)$ and the integer $M_{\Gamma(J)}$ by $M_{J}$. We will also write $\nu_{J}$ instead of $\nu_{\Gamma(J)}$ and we will also refer to $\nu_{J}$ as the Newton filtration induced by $J$.

Definition 4.1. Let us fix a convenient Newton polyhedron $\Gamma_{+} \subseteq \mathbb{R}_{\geqslant 0}^{n}$. If $h \in \mathcal{O}_{n}$ and $h=\sum_{k} a_{k} x^{k}$ is the Taylor expansion of $h$ around the origin, then we define the principal part of $h$ with respect to $\Gamma_{+}$, denoted by $\mathrm{p}_{\Gamma}(h)$, as the polynomial obtained as the sum of all terms $a_{k} x^{k}$ such that $\nu_{\Gamma}(h)=\nu_{\Gamma}\left(x^{k}\right)$. If $\Delta$ is a compact face of $\Gamma_{+}$, we define the principal part of $h$ over $\Delta$, denoted by $\mathrm{p}_{\Gamma, \Delta}(h)$, as the sum of all terms $a_{k} x^{k}$ such that $k \in C(\Delta)$ and $\nu_{\Gamma}(h)=\nu_{\Gamma}\left(x^{k}\right)$. If no such terms exist, then we set $\mathrm{p}_{\Gamma, \Delta}(h)=0$. When there is no risk of confusion, then we denote $\mathrm{p}_{\Gamma, \Delta}(h)$ simply by $\mathrm{p}_{\Delta}(h)$.

Let us fix a monomial ideal $J$ of $\mathcal{O}_{n}$ of finite colength. Let $\Delta$ be a compact face of $\Gamma_{+}(J)$. If $h \in \mathcal{O}_{n}$, then we denote the polynomials $\mathrm{p}_{\Gamma(J)}(h)$ and $\mathrm{p}_{\Gamma(J), \Delta}(h)$ by $\mathrm{p}_{J}(h)$ and $\mathrm{p}_{J, \Delta}(h)$, respectively. If $I$ is an arbitrary ideal of $\mathcal{O}_{n}$, then we define the ideals:

$$
\begin{aligned}
\mathrm{p}_{J, \Delta}(I) & =\left\langle\mathrm{p}_{J, \Delta}(h): h \in I, \nu_{J}(h)=\nu_{J}(I)\right\rangle \\
I_{C(\Delta)} & =\left\langle h_{C(\Delta)}: h \in I\right\rangle .
\end{aligned}
$$

Given a polynomial $h \in \mathbb{C}\left[x_{1}, \ldots, x_{n}\right]$, we say that $h$ is $J$-homogeneous when $\nu_{J}\left(x^{k}\right)=$ $\nu_{J}(h)$, for all $k \in \operatorname{supp}(h)$. If $H=\left(h_{1}, \ldots, h_{p}\right):\left(\mathbb{C}^{n}, 0\right) \rightarrow\left(\mathbb{C}^{p}, 0\right)$ is a polynomial map, then we say that $H$ is $J$-homogeneous when $h_{i}$ is $J$-homogeneous, for all $i=1, \ldots, p$.

We denote the $n$-dimensional volume of a compact set $K \subseteq \mathbb{R}^{n}$ by $\mathrm{V}_{n}(K)$. Joining [7, Theorem 3.3] and [5, Proposition 3.5, Corollary 3.8], we have the following result.

Theorem 4.2. Let $g=\left(g_{1}, \ldots, g_{n}\right):\left(\mathbb{C}^{n}, 0\right) \rightarrow\left(\mathbb{C}^{n}, 0\right)$ be an analytic map germ such that $g^{-1}(0)=\{0\}$. Let $\Gamma_{+} \subseteq \mathbb{R}_{\geqslant 0}^{n}$ be a convenient Newton polyhedron, let $d_{i}=\nu_{\Gamma}\left(g_{i}\right)$, for all 
$i=1, \ldots, n$. Then

$$
\operatorname{dim}_{\mathbb{C}} \frac{\mathcal{O}_{n}}{\left\langle g_{1}, \ldots, g_{n}\right\rangle} \geqslant \frac{d_{1} \cdots d_{n}}{M_{\Gamma}^{n}} n ! \mathrm{V}_{n}\left(\Gamma_{-}\right)
$$

and the following conditions are equivalent:

(a) equality holds in (10);

(b) for each compact facet $\Delta$ of $\Gamma_{+}$, the ideal of $\mathcal{R}_{\Delta}$ generated by $\mathrm{p}_{\Delta}\left(g_{1}\right), \ldots, \mathrm{p}_{\Delta}\left(g_{n}\right)$ has finite colength in $\mathcal{R}_{\Delta}$;

(c) the set germ at 0 of common zeros of $\mathrm{p}_{\Delta}\left(g_{1}\right), \ldots, \mathrm{p}_{\Delta}\left(g_{n}\right)$ is contained in $\left\{x \in \mathbb{C}^{n}\right.$ : $\left.x_{1} \cdots x_{n}=0\right\}$, for all compact faces $\Delta$ of $\Gamma_{+}$.

If $I$ is a non-zero ideal of $\mathcal{O}_{n}$, then we define the order of $I$, denoted by $\operatorname{ord}(I)$, as $\operatorname{ord}(I)=\max \left\{r \in \mathbb{Z}_{\geqslant 0}: I \subseteq \mathbf{m}_{n}^{r}\right\}$. We set ord $(0)=\infty$.

It is a well-known fact that if $J$ denotes a monomial ideal of finite colength of $\mathcal{O}_{n}$, then $e(J)=n ! \mathrm{V}_{n}\left(\Gamma_{-}(J)\right)$ (see for instance [22, p. 239]). Let $I_{1}, \ldots, I_{n}$ be ideals of $\mathcal{O}_{n}$ such that $\sigma\left(I_{1}, \ldots, I_{n}\right)<\infty$. By Proposition 2.2 we have that $\sigma\left(I_{1}, \ldots, I_{n}\right)=e\left(g_{1}, \ldots, g_{n}\right)$, for some $\left(g_{1}, \ldots, g_{n}\right) \in I_{1} \oplus \cdots \oplus I_{n}$. In particular $\nu_{J}\left(I_{i}\right) \leqslant \nu_{J}\left(g_{i}\right)$, for all $i=1, \ldots, n$. Hence relation (10) implies that

$$
\sigma\left(I_{1}, \ldots, I_{n}\right) \geqslant \frac{\nu_{J}\left(I_{1}\right) \cdots \nu_{J}\left(I_{n}\right)}{M_{J}^{n}} e(J)
$$

Definition 4.3. Under the conditions of the above paragraph, we say that the $n$-tuple of ideals $\left(I_{1}, \ldots, I_{n}\right)$ is $J$-non-degenerate when equality holds in (11).

We will denote the term of the right hand side of (11) by $A_{J}\left(I_{1}, \ldots, I_{n}\right)$. We remark that $A_{\mathbf{m}_{n}}\left(I_{1}, \ldots, I_{n}\right)=\operatorname{ord}\left(I_{1}\right) \cdots \operatorname{ord}\left(I_{n}\right)$. If $h \in \mathcal{O}_{n}, h \neq 0$, then we will write $A_{J}\left(h, I_{2}, \ldots, I_{n}\right)$ instead of $A_{J}\left(\langle h\rangle, I_{2}, \ldots, I_{n}\right)$ and we accordingly extend this notation when any other ideal appearing in $A_{J}\left(I_{1}, \ldots, I_{n}\right)$ is principal. In particular, if $g_{1}, \ldots, g_{n}$ are non-zero elements of $\mathcal{O}_{n}$, then we write $A_{J}\left(g_{1}, \ldots, g_{n}\right)$ instead of $A_{J}\left(\left\langle g_{1}\right\rangle, \ldots,\left\langle g_{n}\right\rangle\right)$.

Let $g=\left(g_{1}, \ldots, g_{n}\right):\left(\mathbb{C}^{n}, 0\right) \rightarrow\left(\mathbb{C}^{n}, 0\right)$ be an analytic map germ. We denote by $I(g)$ the ideal of $\mathcal{O}_{n}$ generated by the components of $g$. We say that $g$ is $J$-non-degenerate when the $n$-tuple of ideals $\left(\left\langle g_{1}\right\rangle, \ldots,\left\langle g_{n}\right\rangle\right)$ is $J$-non-degenerate.

Proposition 4.4. Let $J$ be a monomial ideal of $\mathcal{O}_{n}$ of finite colength and let $I_{1}, \ldots, I_{n}$ be ideals of $\mathcal{O}_{n}$ such that $\sigma\left(I_{1}, \ldots, I_{n}\right)<\infty$. Then $\left(I_{1}, \ldots, I_{n}\right)$ is $J$-non-degenerate if and only if there exist $a_{1}, \ldots, a_{n}, d \in \mathbb{Z}_{\geqslant 1}$ such that $\sigma\left(I_{1}^{a_{1}}, \ldots, I_{n}^{a_{n}}\right)=e\left(J^{d}\right)$ and $\nu_{J}\left(I_{1}^{a_{1}}\right)=\cdots=$ $\nu_{J}\left(I_{n}^{a_{n}}\right)=d M_{J}$.

Proof. Let $M=M_{J}$. Let us see the only if part. So, let us assume that

$$
\sigma\left(I_{1}, \ldots, I_{n}\right)=\frac{d_{1} \cdots d_{n}}{M^{n}} e(J)
$$


where $d_{i}=\nu_{J}\left(I_{i}\right)$, for all $i=1, \ldots, n$. Let us define $d=d_{1} \cdots d_{n}$ and $a_{i}=\frac{d}{d_{i}} M$, for all $i=1, \ldots, n$. Clearly we have $\nu_{J}\left(I_{i}^{a_{i}}\right)=a_{i} \nu_{J}\left(I_{i}\right)=\frac{d}{d_{i}} M d_{i}=d M$, for all $i=1, \ldots, n$, and

$$
\sigma\left(I_{1}^{a_{1}}, \ldots, I_{n}^{a_{n}}\right)=a_{1} \cdots a_{n} \sigma\left(I_{1}, \ldots, I_{n}\right)=\frac{d^{n}}{d} M^{n} \frac{d_{1} \cdots d_{n}}{M^{n}} e(J)=d^{n} e(J)=e\left(J^{d}\right) .
$$

Let us see the if part. Let $a_{1}, \ldots, a_{n}, d \in \mathbb{Z}_{\geqslant 1}$ such that $\sigma\left(I_{1}^{a_{1}}, \ldots, I_{n}^{a_{n}}\right)=e\left(J^{d}\right)$ and $\nu_{J}\left(I_{1}^{a_{1}}\right)=\cdots=\nu_{J}\left(I_{n}^{a_{n}}\right)=d M_{J}$. In particular $\nu_{J}\left(I_{i}\right)=\frac{d}{a_{i}} M$, for all $i=1, \ldots, n$.

The equality $\sigma\left(I_{1}^{a_{1}}, \ldots, I_{n}^{a_{n}}\right)=e\left(J^{d}\right)$ is equivalent to saying that $a_{1} \cdots a_{n} \sigma\left(I_{1}, \ldots, I_{n}\right)=$ $d^{n} e(J)$. Therefore

$$
\sigma\left(I_{1}, \ldots, I_{n}\right)=\frac{d^{n}}{a_{1} \cdots a_{n}} M^{n} \frac{1}{M^{n}} e(J)=\frac{\nu_{J}\left(I_{1}\right) \cdots \nu_{J}\left(I_{n}\right)}{M^{n}} e(J) .
$$

Hence the result follows.

As we will see in Theorem 4.10, the $J$-non-degeneracy of sequences $\left(I_{1}, \ldots, I_{n}\right)$ of monomial ideals admits a combinatorial characterization.

Proposition 4.5. Let $J$ be a monomial ideal of $\mathcal{O}_{n}$ of finite colength and let $g:\left(\mathbb{C}^{n}, 0\right) \rightarrow$ $\left(\mathbb{C}^{n}, 0\right)$ be a complex analytic map such that $g^{-1}(0)=\{0\}$. Let $a_{1}, \ldots, a_{n}, d \in \mathbb{Z}_{\geqslant 1}$. Then the following conditions are equivalent.

(a) $e\left(g_{1}^{a_{1}}, \ldots, g_{n}^{a_{n}}\right)=e\left(J^{d}\right)$ and $\nu_{J}\left(g_{1}^{a_{1}}\right)=\cdots=\nu_{J}\left(g_{n}^{a_{n}}\right)=d M_{J}$.

(b) $\overline{\left\langle g_{1}^{a_{1}}, \ldots, g_{n}^{a_{n}}\right\rangle}=\overline{J^{d}}$.

Proof. Let $M=M_{J}$. Let us suppose that (a) holds. The condition $\nu_{J}\left(g_{1}^{a_{1}}\right)=\cdots=\nu_{J}\left(g_{n}^{a_{n}}\right)=$ $d M$ implies that $g_{i}^{a_{i}} \in \overline{J^{d}}$, for all $i=1, \ldots, n$. Then relation (b) follows, by the Rees' multiplicity theorem [12, p. 222].

Let us now assume that (b) holds. Then the relation $e\left(g_{1}^{a_{1}}, \ldots, g_{n}^{a_{n}}\right)=e\left(J^{d}\right)$ follows automatically, since the Samuel multiplicity is invariant by integral closures.

Since $J^{d}$ is a monomial ideal of finite colength, there exists at least one compact face $\Delta$ of $\Gamma_{+}\left(J^{d}\right)$ of dimension $n-1$. Let $\mathcal{R}_{\Delta}$ be the subring of $\mathcal{O}_{n}$ given by all $h \in \mathcal{O}_{n}$ whose support is contained in $C(\Delta)$. The equality (b) implies that the ideal $\left\langle g_{1}^{a_{1}}, \ldots, g_{n}^{a_{n}}\right\rangle$ is a reduction of $\overline{J^{d}}$, which is to say that $\left\langle g_{1}^{a_{1}}, \ldots, g_{n}^{a_{n}}\right\rangle$ is Newton non-degenerate and $\Gamma_{+}\left(g_{1}^{a_{1}}, \ldots, g_{n}^{a_{n}}\right)=$ $\Gamma_{+}\left(J^{d}\right)$ (see [7, Theorem 2.11] for the characterization of reductions of monomial ideals). In particular, the ideal of $\mathcal{R}_{\Delta}$ generated by $\left(g_{1}^{a_{1}}\right)_{\Delta}, \ldots,\left(g_{n}^{a_{n}}\right)_{\Delta}$ has finite colength in $\mathcal{R}_{\Delta}$. Hence $\left(g_{i}^{a_{i}}\right)_{\Delta} \neq 0$, for all $i=1, \ldots, n$, since $\operatorname{dim} \mathcal{R}_{\Delta}=n$. In particular, this says that $\nu_{J}\left(g_{i}^{a_{i}}\right)=d M$, for all $i=1, \ldots, n$, and then (a) follows.

For the sake of completeness we add the following consequence of Propositions 4.4 and 4.5.

Corollary 4.6. Let $J$ be a monomial ideal of $\mathcal{O}_{n}$ of finite colength and let $g:\left(\mathbb{C}^{n}, 0\right) \rightarrow$ $\left(\mathbb{C}^{n}, 0\right)$ be a complex analytic map such that $g^{-1}(0)=\{0\}$. Then $g$ is $J$-non-degenerate if and only if there exist $a_{1}, \ldots, a_{n}, d \in \mathbb{Z}_{\geqslant 1}$ such that $\overline{\left\langle g_{1}^{a_{1}}, \ldots, g_{n}^{a_{n}}\right\rangle}=\overline{J^{d}}$. 
Proof. By Proposition 4.4, the map $g$ is $J$-non-degenerate if and only if there exist some $a_{1}, \ldots, a_{n}, d \in \mathbb{Z}_{\geqslant 1}$ such that $\sigma\left(\left\langle g_{1}^{a_{1}}\right\rangle, \ldots,\left\langle g_{n}^{a_{n}}\right\rangle\right)=e\left(J^{d}\right)$ and $\nu_{J}\left(g_{1}^{a_{1}}\right)=\cdots=\nu_{J}\left(g_{n}^{a_{n}}\right)=d M_{J}$. Since $\sigma\left(\left\langle g_{1}^{a_{1}}\right\rangle, \ldots,\left\langle g_{n}^{a_{n}}\right\rangle\right)=e\left(g_{1}^{a_{1}}, \ldots, g_{n}^{a_{n}}\right)$ (see Proposition 2.2), then the result follows from Proposition 4.5 .

Corollary 4.6 motivates the following definition in the context of an arbitrary Noetherian local ring.

Definition 4.7. Let $(R, \mathbf{m})$ be a Noetherian local ring and let $J$ be a proper ideal of $\mathcal{O}_{n}$. Let $g_{1}, \ldots, g_{n}$ be a sequence of non-zero elements of $R$. We say that the $n$-tuple $\left(g_{1}, \ldots, g_{n}\right)$ is $J$-non-degenerate if and only if there exists some $a_{1}, \ldots, a_{n}, d \in \mathbb{Z}_{\geqslant 1}$ such that $\overline{\left\langle g_{1}^{a_{1}}, \ldots, g_{n}^{a_{n}}\right\rangle}=\overline{J^{d}}$.

It is interesting to remark that if $g:\left(\mathbb{C}^{n}, 0\right) \rightarrow\left(\mathbb{C}^{n}, 0\right)$ is $J$-non-degenerate, where $J$ is a monomial ideal of $\mathcal{O}_{n}$ of finite colength, then the sequence of mixed multiplicities $e_{i}(I(g), J)$, $i=1, \ldots, n$, is determined by the Newton filtration induced by $J$.

Proposition 4.8. Let $J$ be a monomial ideal of $\mathcal{O}_{n}$ of finite colength and let $g=\left(g_{1}, \ldots, g_{n}\right)$ : $\left(\mathbb{C}^{n}, 0\right) \rightarrow\left(\mathbb{C}^{n}, 0\right)$ be a J-non-degenerate map. Let $d_{i}=\nu_{J}\left(g_{i}\right)$, for all $i=1, \ldots, n$. Let us suppose that $d_{1} \leqslant \cdots \leqslant d_{n}$. Then

$$
e_{i}(I(g), J)=\frac{d_{1} \cdots d_{i}}{M^{i}} e(J)
$$

Proof. Let $I=I(\mathrm{~g})$. By the Theorem of existence of joint reductions (see [18, p. 4] or [12, p. 336]), there exist a sufficiently general element $\left(f_{1}, \ldots, f_{i}, f_{i+1}, \ldots, f_{n}\right)$ of $I \oplus \cdots \oplus I \oplus J \oplus$ $\cdots \oplus J$ such that

$$
e_{i}(I, J)=e(I, \ldots, I, J, \ldots, J)=e\left(f_{1}, \ldots, f_{i}, f_{i+1}, \ldots, f_{n}\right) .
$$

Since $f_{j}$ is a generic linear combination of $g_{1}, \ldots, g_{n}$, for all $j=1, \ldots, i$, we observe that

$$
\left\langle f_{1}, \ldots, f_{i}\right\rangle=\left\langle g_{1}+\sum_{\ell=i+1}^{n} \alpha_{1 \ell} g_{\ell}, \ldots, g_{i}+\sum_{\ell=i+1}^{n} \alpha_{i \ell} g_{\ell}\right\rangle,
$$

for some constants $\alpha_{j \ell} \in \mathbb{C}, j=1, \ldots, i, \ell=i+1, \ldots, n$. Let $h_{j}=g_{j}+\sum_{\ell=i+1}^{n} \alpha_{j \ell} g_{\ell}$, for all $j=1, \ldots, i$.

By appropriately taking the coefficients $\alpha_{j \ell}$, by virtue of Theorem 4.2 , we obtain that $\left(h_{1}, \ldots, h_{i}, f_{i+1}, \ldots, f_{n}\right)$ is $J$-non-degenerate with $\nu_{J}\left(h_{j}\right)=\nu_{J}\left(g_{j}\right)=d_{j}$, for all $j=1, \ldots, i$, and $\nu_{J}\left(f_{j}\right)=M$, for all $j=i+1, \ldots, n$. Hence, by (12) and (13) we obtain that

$$
e_{i}(I, J)=e\left(h_{1}, \ldots, h_{i}, f_{i+1}, \ldots, f_{n}\right)=\frac{d_{1} \cdots d_{i} M^{n-i}}{M^{n}} e(J)=\frac{d_{1} \cdots d_{i}}{M^{i}} e(J) .
$$

Remark 4.9. Under the hypothesis of Proposition 4.8, we observe that the condition

$$
e_{0}(I(g), J)=e_{1}(I(g), J)=\cdots=e_{n}(I(g), J)
$$


is equivalent to saying that $d_{1}=\cdots=d_{n}=M$, which in turn is equivalent to the condition $\overline{\left\langle g_{1}, \ldots, g_{n}\right\rangle}=\bar{J}$, by Proposition 4.5 . Then we recovered a particular case of the result of Teissier characterizing the equality $\bar{I}=\bar{J}$ when no inclusion relation between $I$ and $J$ is assumed (see [20, Théorème 4.2, p. 341]).

\subsection{Characterization of $J$-non-degeneracy of sequences of monomial ideals}

Let $I_{1}, \ldots, I_{n}$ be monomial ideals of $\mathcal{O}_{n}$ such that $\sigma\left(I_{1}, \ldots, I_{n}\right)<\infty$. We denote by $\mathcal{S}\left(I_{1}, \ldots, I_{n}\right)$ the family of maps $g=\left(g_{1}, \ldots, g_{n}\right):\left(\mathbb{C}^{n}, 0\right) \rightarrow\left(\mathbb{C}^{n}, 0\right)$ for which $g^{-1}(0)=$ $\{0\}, g_{i} \in I_{i}$, for all $i=1, \ldots, n$, and $\sigma\left(I_{1}, \ldots, I_{n}\right)=e\left(g_{1}, \ldots, g_{n}\right)$. The elements of $\mathcal{S}\left(I_{1}, \ldots, I_{n}\right)$ were characterized in [2, Theorem 3.10]. We denote by $\mathcal{S}_{0}\left(I_{1}, \ldots, I_{n}\right)$ the maps $g \in \mathcal{S}\left(I_{1}, \ldots, I_{n}\right)$ for which $\Gamma_{+}\left(g_{i}\right)=\Gamma_{+}\left(I_{i}\right)$, for all $i=1, \ldots, n$.

Theorem 4.10. Let $I_{1}, \ldots, I_{n}, J$ be monomial ideals of $\mathcal{O}_{n}$ such that $\sigma\left(I_{1}, \ldots, I_{n}\right)<\infty$ and $J$ has finite colength. Then the following conditions are equivalent:

(a) $\left(I_{1}, \ldots, I_{n}\right)$ is $J$-non-degenerate.

(b) $\left(g_{1}, \ldots, g_{n}\right)$ is $J$-non-degenerate, for every $\left(g_{1}, \ldots, g_{n}\right) \in \mathcal{S}\left(I_{1}, \ldots, I_{n}\right)$;

(c) $\left(g_{1}, \ldots, g_{n}\right)$ is J-non-degenerate, for some $\left(g_{1}, \ldots, g_{n}\right) \in \mathcal{S}_{0}\left(I_{1}, \ldots, I_{n}\right)$;

(d) for any compact face $\Delta$ of $\Gamma_{+}(J)$ we have

$$
\left|\left\{i: \mathrm{p}_{J, \Delta}\left(I_{i}\right) \neq 0\right\}\right| \geqslant \operatorname{dim}(\Delta)+1 \text {. }
$$

Proof. Let $M=M_{J}$. As remarked before relation (11), given an element $\left(g_{1}, \ldots, g_{n}\right) \in$ $\mathcal{S}\left(I_{1}, \ldots, I_{n}\right)$, we have that

$$
\sigma\left(I_{1}, \ldots, I_{n}\right)=e\left(g_{1}, \ldots, g_{n}\right) \geqslant \frac{\nu_{J}\left(g_{1}\right) \cdots \nu_{J}\left(g_{n}\right)}{M^{n}} e(J) \geqslant A_{J}\left(I_{1}, \ldots, I_{n}\right) .
$$

Let is prove $(\mathrm{a}) \Rightarrow(\mathrm{b})$. So, let us suppose that $\sigma\left(I_{1}, \ldots, I_{n}\right)=A_{J}\left(I_{1}, \ldots, I_{n}\right)$. If $\left(g_{1}, \ldots, g_{n}\right) \in \mathcal{S}\left(I_{1}, \ldots, I_{n}\right)$, then 15$)$ shows that $e\left(g_{1}, \ldots, g_{n}\right)=A_{J}\left(g_{1}, \ldots, g_{n}\right)$, which is to say that $g$ is $J$-non-degenerate.

The implication $(\mathrm{b}) \Rightarrow(\mathrm{c})$ is obvious. Let us prove $(\mathrm{c}) \Rightarrow(\mathrm{d})$. Let $\left(g_{1}, \ldots, g_{n}\right) \in$ $\mathcal{S}_{0}\left(I_{1}, \ldots, I_{n}\right)$ such that $\left(g_{1}, \ldots, g_{n}\right)$ is $J$-non-degenerate and let us fix a compact face $\Delta$ of $\Gamma_{+}(J)$. Let $r=\operatorname{dim} \Delta$. The ideal of $\mathcal{R}_{\Delta}$ generated by $\mathrm{p}_{\Delta}\left(g_{1}\right), \ldots, \mathrm{p}_{\Delta}\left(g_{n}\right)$ has finite colength in $\mathcal{R}_{\Delta}$, by Theorem 4.2. Since $\operatorname{dim} \mathcal{R}_{\Delta}=r+1$, at least $r+1$ elements of $\left\{\mathrm{p}_{\Delta}\left(g_{1}\right), \ldots, \mathrm{p}_{\Delta}\left(g_{n}\right)\right\}$ are not zero. By definition, the condition $\left(g_{1}, \ldots, g_{n}\right) \in \mathcal{S}_{0}\left(I_{1}, \ldots, I_{n}\right)$ implies that $\Gamma_{+}\left(g_{i}\right)=\Gamma_{+}\left(I_{i}\right)$, for all $i=1, \ldots, n$. Thus $\nu_{J}\left(g_{i}\right)=\nu_{J}\left(I_{i}\right)$, for all $i=1, \ldots, n$. Then condition (d) follows, by the definition of the ideals $\mathrm{p}_{J, \Delta}\left(I_{1}\right), \ldots, \mathrm{p}_{J, \Delta}\left(I_{n}\right)$ (see (8)).

Let us prove $(\mathrm{d}) \Rightarrow(\mathrm{a})$. Let $G_{i}$ be a fixed minimal generating system of $I_{i}$ formed by monomials, for all $i=1, \ldots, n$, and let $\mathbf{G}=\left(G_{1}, \ldots, G_{n}\right)$. Let us fix a compact face $\Delta$ of $\Gamma_{+}(J)$ and let $r=\operatorname{dim}(\Delta)$. By hypothesis, there exist a set of indices $B_{\Delta} \subseteq\{1, \ldots, n\}$ such that $\left|B_{\Delta}\right|=r+1$ and $\mathrm{p}_{J, \Delta}\left(I_{j}\right) \neq 0$, for all $j \in B_{\Delta}$. Let us write $B_{\Delta}=\left\{j_{\Delta, 1}, \ldots, j_{\Delta, r+1}\right\}$, for some $1 \leqslant j_{\Delta, 1}<\cdots<j_{\Delta, r+1} \leqslant n$. Let us denote by $G_{j}^{\Delta}$ be the set of monomials $x^{k}$ of $G_{j}$ such that $k \in C(\Delta)$ and $\phi_{J}(k)=\nu_{J}\left(I_{j}\right)$, for all $j=1, \ldots, n$. By the definition of $B_{\Delta}$, we have that $G_{j}^{\Delta} \neq \emptyset$, for all $j \in B_{\Delta}$. Let $\mathbf{G}^{\Delta}=\left(G_{j_{\Delta, 1}}^{\Delta}, \ldots, G_{j_{\Delta, r+1}}^{\Delta}\right)$. 
As in the proof of Theorem 3.2, we can apply Theorem 2.3 to the $(r+1)$-tuple of ideals $\left(\mathrm{p}_{J, \Delta}\left(I_{j_{\Delta, 1}}\right), \ldots, \mathrm{p}_{J, \Delta}\left(I_{j_{\Delta, r+1}}\right)\right)$. That is, let us identify the set of $\mathbf{G}^{\Delta}$-maps with $\mathbb{C}^{N}$, where $N=\left|G_{j_{\Delta, 1}}^{\Delta}\right|+\cdots+\left|G_{j_{\Delta, r+1}}^{\Delta}\right|$, via the vector of coefficients of the $\mathbf{G}^{\Delta}$-maps. Then, there exist a Zariski-open subset $U_{\Delta} \subseteq \mathbb{C}^{N}$, such that any $\mathbf{G}^{\Delta}$-map whose vector of coefficients belongs to $U_{\Delta}$ is a joint reduction of $\left(\mathrm{p}_{J, \Delta}\left(I_{j_{\Delta, 1}}\right), \ldots, \mathrm{p}_{J, \Delta}\left(I_{j_{\Delta, r+1}}\right)\right)$.

Since $\Gamma_{+}(J)$ has a finite number of compact faces, the above discussion shows that there exists a G-map $\left(g_{1}, \ldots, g_{n}\right):\left(\mathbb{C}^{n}, 0\right) \rightarrow\left(\mathbb{C}^{n}, 0\right)$ with the property that, for any compact face $\Delta$ of $\Gamma_{+}(J)$, the map $\left(\mathrm{p}_{J, \Delta}\left(g_{j_{\Delta, 1}}\right), \ldots, \mathrm{p}_{J, \Delta}\left(g_{j_{\Delta, \operatorname{dim}(\Delta)+1}}\right)\right)$ is a joint reduction of $\left(\mathrm{p}_{J, \Delta}\left(I_{j_{\Delta, 1}}\right), \ldots, \mathrm{p}_{J, \Delta}\left(I_{j_{\Delta, \operatorname{dim}(\Delta)+1}}\right)\right)$.

Let us fix a compact face $\Delta$ of $\Gamma_{+}(J)$ and let $r=\operatorname{dim}(\Delta)$. In order to simplify the notation, let us suppose that $B_{\Delta}=\{1, \ldots, r+1\}$. Then, by (6), we obtain that

$$
\overline{\mathrm{p}_{J, \Delta}\left(g_{1}\right) \mathrm{p}_{\Delta}\left(I_{2}\right) \cdots \mathrm{p}_{\Delta}\left(I_{r+1}\right)+\cdots+\mathrm{p}_{J, \Delta}\left(g_{r+1}\right) \mathrm{p}_{\Delta}\left(I_{1}\right) \cdots \mathrm{p}_{\Delta}\left(I_{r}\right)}=\overline{\mathrm{p}_{\Delta}\left(I_{1}\right) \cdots \mathrm{p}_{\Delta}\left(I_{r+1}\right)} .
$$

In particular,

$$
\overline{\mathrm{p}_{\Delta}\left(I_{1}\right) \cdots \mathrm{p}_{\Delta}\left(I_{r+1}\right)} \subseteq \overline{\left\langle\mathrm{p}_{J, \Delta}\left(g_{1}\right), \ldots, \mathrm{p}_{J, \Delta}\left(g_{r+1}\right)\right\rangle}
$$

and this implies that

$$
\mathbf{V}\left(\mathrm{p}_{J, \Delta}\left(g_{1}\right), \ldots, \mathrm{p}_{J, \Delta}\left(g_{r+1}\right)\right) \subseteq \mathbf{V}\left(\mathrm{p}_{\Delta}\left(I_{1}\right) \cdots \mathrm{p}_{\Delta}\left(I_{r+1}\right)\right) \subseteq \mathbf{H}
$$

where the last inclusion is a consequence of the fact that $\mathrm{p}_{\Delta}\left(I_{1}\right) \cdots \mathrm{p}_{\Delta}\left(I_{r+1}\right)$ are monomial ideals of $\mathcal{O}_{n}$.

Therefore, we conclude that, for each compact face $\Delta$ of $\Gamma_{+}(J)$, the set of common zeros of the polynomials $\mathrm{p}_{J, \Delta}\left(g_{1}\right), \ldots, \mathrm{p}_{J, \Delta}\left(g_{n}\right)$ is contained in $\mathbf{H}$, which means that $\left(g_{1}, \ldots, g_{n}\right)$ is $J$-non-degenerate, by virtue of Theorem 4.2. Then, all inequalities of (15) become equalities. Hence (a) holds and we have completed the proof.

Corollary 4.11. Let $I_{1}, \ldots, I_{n}$ be monomial ideals of $\mathcal{O}_{n}$ such that $\sigma\left(I_{1}, \ldots, I_{n}\right)<\infty$. Then $\sigma\left(I_{1}, \ldots, I_{n}\right) \geqslant \operatorname{ord}\left(I_{1}\right) \cdots \operatorname{ord}\left(I_{n}\right)$ and equality holds if and only if for all non-empty $\mathrm{L} \subseteq\{1, \ldots, n\}$ we have that

$$
\left|\left\{i: \operatorname{ord}\left(I_{i}^{\mathrm{L}}\right)=\operatorname{ord}\left(I_{i}\right)\right\}\right| \geqslant|\mathrm{L}| \text {. }
$$

Proof. Let $\mathbf{m}=\mathbf{m}_{n}$. Let us apply Theorem 4.10 in the case $J=\mathbf{m}$. The filtrating map $\phi_{\mathbf{m}}: \mathbb{R}_{\geqslant 0}^{n} \rightarrow \mathbb{R}_{\geqslant 0}$ is given by $\phi_{\mathbf{m}}(k)=|k|$, for all $k \in \mathbb{R}_{\geqslant 0}^{n}$. Hence $\nu_{\mathbf{m}}(I)=\operatorname{ord}(I)$. Moreover, if $\Delta \subseteq \Gamma_{+}(\mathbf{m})$ and $j \in\{0,1, \ldots, n-1\}$, then $\Delta$ is a compact face of dimension $j$ of $\Gamma_{+}(\mathbf{m})$ if and only if there exists some $\mathrm{L} \subseteq\{1, \ldots, n\}$ such that $|\mathrm{L}|=j+1$ and $\Delta=\Gamma(\mathbf{m})^{\mathrm{L}}$, where we recall that $\Gamma(\mathbf{m})$ denotes the Newton boundary of $\Gamma_{+}(\mathbf{m})$.

Let us fix a non-empty subset $\mathrm{L} \subseteq\{1, \ldots, n\}$ and let $\Delta=\Gamma(\mathbf{m})^{\mathrm{L}}$. If $i \in\{1, \ldots, n\}$, then $\mathrm{p}_{\mathbf{m}, \Delta}\left(I_{i}\right) \neq 0$ if and only if there exists some $k \in \operatorname{supp}\left(I_{i}^{\mathrm{L}}\right)$ such that $|k|=\operatorname{ord}\left(I_{i}\right)$, which is to say that $\operatorname{ord}\left(I_{i}\right)=\operatorname{ord}\left(I_{i}^{\mathrm{L}}\right)$. Then the result follows as a consequence of Theorem 4.10 . 


\section{Central maps With Respect to pairs of ideals}

Given two monomial ideals $I$ and $J$ of $\mathcal{O}_{n}$ of finite colength, it is obvious that it is always possible to find a $J$-non-degenerate $n$-tuple $\left(K_{1}, \ldots, K_{n}\right)$ of monomial ideals contained in $I$. It suffices to take each $K_{i}$ equal to some power of $J$ contained in $I$. However, we will see that, by replacing $I$ by suitable powers $I^{s}$, such an $n$-tuple can be constructed so that the Newton boundary of $\Gamma_{+}\left(K_{i}\right)$ intersects the Newton boundary of $\Gamma_{+}\left(I^{s}\right)$, for all $i=1, \ldots, n$ (roughly speaking this means that the ideals $K_{1}, \ldots, K_{n}$ will not be far away from $I^{s}$ ). This fact will lead to a characterization of when the integral closure of some power of $I$ is equal to the integral closure of the ideal generated by the components of a $J$-non-degenerate map $g=\left(g_{1}, \ldots, g_{n}\right):\left(\mathbb{C}^{n}, 0\right) \rightarrow\left(\mathbb{C}^{n}, 0\right)$

Definition 5.1. Let $I$ and $J$ be monomial ideals of $\mathcal{O}_{n}$ of finite colength. For any $i \in$ $\{1, \ldots, n\}$, we define the following number:

$$
a_{i, J}(I)=\max \left\{\nu_{J}\left(\Gamma_{+}(I) \cap C(\Delta)\right): \Delta \text { is a compact face of } \Gamma_{+}(J) \text { of dimension } n-i\right\},
$$

where we recall that if $A$ is a closed subset of $\mathbb{R}_{\geqslant 0}^{n}$, then $\nu_{J}(A)=\min \left\{\phi_{J}(k): k \in A\right\}$. Therefore $a_{i, J}(I) \in \mathbb{Q}_{>0}$, for all $i=1, \ldots, n$. It easily follows that $a_{1, J}(I) \leqslant \cdots \leqslant a_{n, J}(I)$. We denote the vector $\left(a_{1, J}(I), \ldots, a_{n, J}(I)\right)$ by $\mathbf{a}_{J}(I)$.

Under the conditions of the above definition, since $\Gamma_{+}(I)=\Gamma_{+}(\bar{I})$, it follows immediately that $a_{i, J}(I)=a_{i, J}(\bar{I})$, for all $i=1, \ldots, n$.

Remark 5.2. Let $I$ be a monomial ideal of $\mathcal{O}_{n}$ and let $\mathbf{m}=\mathbf{m}_{n}$. We denote $a_{i, \mathbf{m}}(I)$ simply by $a_{i}(I)$, for all $i=1, \ldots, n$. Since the set of compact faces of $\Gamma_{+}(\mathbf{m})$ is given by $\left\{\Gamma(\mathbf{m})^{\mathrm{L}}: \mathrm{L} \subseteq\{1, \ldots, n\}, \mathrm{L} \neq \emptyset\right\}$ and $\phi_{\mathbf{m}}(k)=|k|$, for all $k \in \mathbb{R}_{\geqslant 0}^{n}$, then

$$
a_{i}(I)=\max \left\{\operatorname{ord}\left(I^{\mathrm{L}}\right): \mathrm{L} \subseteq\{1, \ldots, n\},|\mathrm{L}|=n-i+1\right\}
$$

and we recover the definition of the integers $a_{i}(I)$ given in [4, p. 197], which in turn was motivated by the expression for the sequence of mixed Łojasiewicz exponents of $I$ given in [6. Corollary 4.2]. We will show some connections between the numbers $a_{i, J}(I)$ and mixed Łojasiewicz exponents (in the sense of [3, 5, 11]) in a subsequent work.

Let $I$ and $J$ be monomial ideals of $\mathcal{O}_{n}$ of finite colength and let $u \in \mathbb{Z}_{\geqslant 0}^{n}, u \neq 0$. We denote by $k_{u}^{I}$ the point of intersection of $\Gamma(I)$ with the half-line $\left\{\lambda u: \lambda \in \mathbb{R}_{\geqslant 0}\right\}$. Therefore

$$
a_{n, J}(I)=\max \left\{\phi_{J}\left(k_{u}^{I}\right): u \in \mathbf{v}\left(\Gamma_{+}(J)\right)\right\} .
$$

We also observe that, under the conditions of Definition 5.1, the maximum that leads to the computation of $a_{i, J}(I)$ is attained at some point of $\mathbf{v}\left(\Gamma_{+}(I)\right) \cup\left\{k_{u}^{I}: u \in \mathbf{v}\left(\Gamma_{+}(J)\right)\right\}$.

The point $k_{u}^{I}$ has rational coordinates, for all $u \in \mathbb{Z}_{\geqslant 0}^{n}, u \neq 0$. So, we define

$$
c_{J}(I)=\min \left\{c \in \mathbb{Z}_{\geqslant 1}: c k_{u}^{I} \in \mathbb{Z}_{\geqslant 0}^{n} \text {, for all } u \in \mathbf{v}\left(\Gamma_{+}(J)\right)\right\}
$$


Theorem 5.3. Let $I$ and $J$ be monomial ideals of $\mathcal{O}_{n}$ of finite colength. Let $c=c_{J}(I)$ and let $M=M_{J}$. For any $i \in\{1, \ldots, n\}$, let us consider the ideal

$$
K_{i}=\left\langle x^{k}: k \in \operatorname{supp}\left(\overline{I^{c M}}\right), \phi_{J}(k)=a_{i, J}\left(\overline{I^{c M}}\right)\right\rangle .
$$

Then $\left(K_{1}, \ldots, K_{n}\right)$ is $J$-non-degenerate.

Proof. Let $\Gamma_{+}=\Gamma_{+}(J)$ and let $\phi=\phi_{J}$. We recall that $c \Gamma_{+}(I)=\Gamma_{+}\left(I^{c}\right)$ and hence $c \Gamma(I)=$ $\Gamma\left(I^{c}\right)$. Thus $c k_{u}^{I}$ is the point where $\Gamma\left(I^{c}\right)$ meets the half-line $\left\{\lambda u: \lambda \in \mathbb{R}_{\geqslant 0}\right\}$, for all $u \in$ $\mathbf{v}\left(\Gamma_{+}(J)\right)$. Let us remark that $c k_{u}^{I}$ has integer coordinates, for all $u \in \mathbf{v}\left(\Gamma_{+}(J)\right)$.

Let us define the ideal $K=\overline{I^{c M}}$. Hence $\operatorname{supp}(K)=\left(c M \Gamma_{+}(I)\right) \cap \mathbb{Z}_{\geqslant 0}^{n}$. Let us remark that, since $a_{i, J}(I)$ is attained at some point belonging to $\mathbf{v}\left(\Gamma_{+}(I)\right) \cup\left\{k_{u}^{I}: u \in \mathbf{v}\left(\Gamma_{+}(J)\right)\right\}$, then $a_{i, J}(K)=c M a_{i, J}(I) \in \mathbb{Z}_{\geqslant 1}$, for all $i=1, \ldots, n$. Moreover, by the definition of $c$ we have that, if $\Delta$ denotes any compact face of $\Gamma_{+}(J)$, then the intersection of $C(\Delta)$ with $\Gamma_{+}(K)$ has integer vertices. Hence $\nu_{J}\left(K_{C(\Delta)}\right)=\nu_{J}\left(\Gamma_{+}(K) \cap C(\Delta)\right)$, where by $(9)$ and the fact that $K$ is integrally closed, the ideal $K_{C(\Delta)}$ is generated by the monomials $x^{k}$ such that $k \in \Gamma_{+}(K) \cap C(\Delta)$.

From the definition of $K_{i}$ we obtain that $\nu_{J}\left(K_{i}\right)=a_{i, J}(K)=c M a_{i, J}(I)$, for all $i=1, \ldots, n$. In order to prove the result we will check that condition (d) of Theorem 4.10 applied to $\left(K_{1}, \ldots, K_{n}\right)$ holds. Let $\Delta$ be a compact face of $\Gamma_{+}$and let $r=\operatorname{dim}(\Delta), r \in\{0,1, \ldots, n-1\}$. Then the objective is to prove the inequality $\left|\left\{i: \mathrm{p}_{J, \Delta}\left(K_{i}\right) \neq 0\right\}\right| \geqslant r+1$.

Let $i \in\{n-r, \ldots, n\}$. Then $r \geqslant n-i$, and this implies that there exists some face $\Delta^{\prime} \subseteq \Delta$ of dimension $n-i$.

From the definition of $a_{i, J}(K)$, we have that $\nu_{J}\left(K_{C\left(\Delta^{\prime}\right)}\right)=\nu_{J}\left(\Gamma_{+}(K) \cap C\left(\Delta^{\prime}\right)\right) \leqslant a_{i, J}(K)$. Let us consider a point $k \in \operatorname{supp}\left(K_{C\left(\Delta^{\prime}\right)}\right)$ such that $\phi(k)=\nu_{J}\left(K_{C\left(\Delta^{\prime}\right)}\right)$. By the definition of $K$, it follows that $\nu_{J}\left(K_{C\left(\Delta^{\prime}\right)}\right)=c M \nu_{J}\left(\Gamma_{+}(I) \cap C\left(\Delta^{\prime}\right)\right)$. Let $u$ be any vertex of $\Delta^{\prime}$ and let us consider a point $k^{\prime} \in \mathbb{R}_{\geqslant 0}^{n}$ of the form $k^{\prime}=k+\gamma u$, for some $\gamma \in \mathbb{R}_{\geqslant 0}$. Since $k$ and $u$ belong to the same cone $C\left(\Delta^{\prime}\right)$, then $\phi(k+\gamma u)=\phi(k)+\phi(\gamma u)$. Hence we obtain the following equivalences

$$
\begin{aligned}
\nu_{J}\left(x^{k^{\prime}}\right)=a_{i, J}(K) & \Longleftrightarrow \phi(k+\gamma u)=a_{i, J}(K) \\
& \Longleftrightarrow \phi(k)+\gamma M=c M a_{i, J}(I) \\
& \Longleftrightarrow \gamma=\frac{c M a_{i, J}(I)-\nu_{J}\left(K_{C\left(\Delta^{\prime}\right)}\right)}{M}=c a_{i, J}(I)-c \nu_{J}\left(\Gamma_{+}(I) \cap C\left(\Delta^{\prime}\right)\right) .
\end{aligned}
$$

Since $\operatorname{dim}\left(\Delta^{\prime}\right)=n-i$, then $\nu_{J}\left(\Gamma_{+}(I) \cap C\left(\Delta^{\prime}\right) \leqslant a_{i, J}(I)\right.$, by the definition $a_{i, J}(I)$. Let us assign to $\gamma$ the value determined in (18). Then $\gamma$ is a non-negative integer. Therefore $k^{\prime} \in \mathbb{Z}_{\geqslant 0}^{n}$. Since $k, u \in C\left(\Delta^{\prime}\right)$ and $k^{\prime}=k+\gamma u$, then $k^{\prime} \in C\left(\Delta^{\prime}\right)$ and thus $x^{k^{\prime}} \in K_{C\left(\Delta^{\prime}\right)} \subseteq$ $K_{C(\Delta)} \subseteq K$. In particular, $k^{\prime} \in \operatorname{supp}(K)$ and, by the definition of $\gamma$, we have that $\nu_{J}\left(x^{k^{\prime}}\right)=$ $a_{i, J}(K)$. This means that $x^{k^{\prime}} \in \mathrm{p}_{J, \Delta}\left(K_{i}\right)$. Hence we have proved that $\mathrm{p}_{J, \Delta}\left(K_{i}\right) \neq 0$, for all $i \in\{n-r, \ldots, n\}$. Therefore $\left|\left\{i: \mathrm{p}_{J, \Delta}\left(K_{i}\right) \neq 0\right\}\right| \geqslant r+1=\operatorname{dim}(\Delta)+1$ and thus $\left(K_{1}, \ldots, K_{n}\right)$ is $J$-non-degenerate, by Theorem 4.10 . 
Corollary 5.4. Let $I$ and $J$ be monomial ideals of $\mathcal{O}_{n}$ of finite colength. Let $c=c_{J}(I)$ and let $M=M_{J}$. Then there exists a J-homogeneous polynomial map $F=\left(F_{1}, \ldots, F_{n}\right)$ : $\left(\mathbb{C}^{n}, 0\right) \rightarrow\left(\mathbb{C}^{n}, 0\right)$ such that $F$ is $J$-non-degenerate, $\nu_{J}\left(F_{i}\right)=a_{i, J}\left(I^{c M}\right)$ and $F_{i} \in \overline{I^{c M}}$, for all $i \in\{1, \ldots, n\}$.

Proof. As in the proof of Theorem 5.3 , let $K=\overline{I^{c M}}$. Let $K_{1}, \ldots, K_{n}$ be the ideals defined in (17). By Theorem 5.3, $\left(K_{1}, \ldots, K_{n}\right)$ is $J$-non-degenerate. Then, by Theorem 4.10, there exists a polynomial map $F=\left(F_{1}, \ldots, F_{n}\right):\left(\mathbb{C}^{n}, 0\right) \rightarrow\left(\mathbb{C}^{n}, 0\right)$ such that $F \in \mathcal{S}_{0}\left(K_{1}, \ldots, K_{n}\right)$, $F$ is $J$-non-degenerate and $\nu_{J}\left(F_{i}\right)=\nu_{J}\left(K_{i}\right)=c M a_{i, J}(I)$, for all $i=1, \ldots, n$. The set $\left\{x^{k}: k \in K, \phi_{J}(k)=a_{i, J}(K)\right\}$ is a generating system of $K_{i}$, for all $i=1, \ldots, n$. Therefore, by Proposition 2.2, we can take each polynomial $F_{i}$ as a generic $\mathbb{C}$-linear combination of this generating system of $K_{i}$. Then we conclude that $F_{i}$ can be taken as a $J$-homogeneous polynomial, for all $i=1, \ldots, n$, and the result follows.

If $F$ is any map satisfying the thesis of Corollary 5.4 , then we say that $F$ is a central map with respect to the pair $(I, J)$.

Theorem 5.5. Let $I, J \subseteq \mathcal{O}_{n}$ be monomial ideals of $\mathcal{O}_{n}$ of finite colength. Let $M=M_{J}$. Then

$$
\frac{e(I)}{e(J)} \leqslant \frac{a_{1, J}(I) \cdots a_{n, J}(I)}{M^{n}}
$$

and the following conditions are equivalent:

(a) equality holds in (19);

(b) there exists a polynomial map $F=\left(F_{1}, \ldots, F_{n}\right):\left(\mathbb{C}^{n}, 0\right) \rightarrow\left(\mathbb{C}^{n}, 0\right)$ and some $s \in$ $\mathbb{Z}_{\geqslant 1}$ such that $F$ is $J$-non-degenerate and $J$-homogeneous, $\nu_{J}\left(F_{i}\right)=s a_{i, J}(I)$, for all $i=1, \ldots, n$, and $\overline{I^{s}}=\overline{\left\langle F_{1}, \ldots, F_{n}\right\rangle}$;

(c) there exists a polynomial map $F=\left(F_{1}, \ldots, F_{n}\right):\left(\mathbb{C}^{n}, 0\right) \rightarrow\left(\mathbb{C}^{n}, 0\right)$ and some $s \in \mathbb{Z}_{\geqslant 1}$ such that $F$ is $J$-non-degenerate and J-homogeneous and $\overline{I^{s}}=\overline{\left\langle F_{1}, \ldots, F_{n}\right\rangle}$;

(d) there exists a polynomial map $g=\left(g_{1}, \ldots, g_{n}\right):\left(\mathbb{C}^{n}, 0\right) \rightarrow\left(\mathbb{C}^{n}, 0\right)$ and some $s \in \mathbb{Z}_{\geqslant 1}$ such that $g$ is $J$-non-degenerate and $\overline{I^{s}}=\overline{\left\langle g_{1}, \ldots, g_{n}\right\rangle}$.

Proof. By Corollary 5.4, there exists a central polynomial map $F:\left(\mathbb{C}^{n}, 0\right) \rightarrow\left(\mathbb{C}^{n}, 0\right)$ with respect to the pair $(I, J)$. Let $c=c_{J}(I)$ and let $K=\overline{I^{c M}}$. Hence $F$ is $J$-non-degenerate and $J$-homogeneous with $\nu_{J}\left(F_{i}\right)=a_{i, J}(K)$, for all $i=1, \ldots, n$. Moreover, $F_{i} \in K$, for all $i=1, \ldots, n$. Hence, if we define the ideals $K_{1}, \ldots, K_{n}$ as in (17), then

$$
\begin{aligned}
\sigma\left(K_{1}, \ldots, K_{n}\right)=e\left(F_{1}, \ldots, F_{n}\right) & =\frac{a_{1, J}(K) \cdots a_{n, J}(K)}{M^{n}} e(J) \\
& =(c M)^{n} \frac{a_{1, J}(I) \cdots a_{n, J}(I)}{M^{n}} e(J) \\
& =c^{n} a_{1, J}(I) \cdots a_{n, J}(I) e(J) .
\end{aligned}
$$


Since $K_{i} \subseteq K=\overline{I^{c M}}$, for all $i=1, \ldots, n$, then $\sigma\left(K_{1}, \ldots, K_{n}\right) \geqslant e(K)=(c M)^{n} e(I)$. Joining this with (20) and (22) we obtain that

$$
c^{n} a_{1, J}(I) \cdots a_{n, J}(I) e(J) \geqslant(c M)^{n} e(I) .
$$

Thus inequality (19) follows.

Let us prove $(\mathrm{a}) \Rightarrow(\mathrm{b})$. If equality holds in $(19)$, then $(20)-(22)$ imply that $e\left(F_{1}, \ldots, F_{n}\right)=$ $e(K)$. Then, by the Rees' Multiplicity Theorem (see [10, p. 147] or [12, p. 222]), we conclude that $\overline{\left\langle F_{1}, \ldots, F_{n}\right\rangle}=\bar{K}=\overline{I^{c M}}=K$ and thus (b) follows by taking $s=c M$.

Let us prove $(\mathrm{b}) \Rightarrow(\mathrm{a})$. If $F=\left(F_{1}, \ldots, F_{n}\right):\left(\mathbb{C}^{n}, 0\right) \rightarrow\left(\mathbb{C}^{n}, 0\right)$ denotes any map satisfying the conditions of (b), for some $s \in \mathbb{Z}_{\geqslant 1}$, then $e\left(F_{1}, \ldots, F_{n}\right)=e\left(I^{s}\right)=s^{n} e(I)$. Moreover $e\left(F_{1}, \ldots, F_{n}\right)=\nu_{J}\left(F_{1}\right) \cdots \nu_{J}\left(F_{n}\right) \frac{e(J)}{M^{n}}=s^{n} a_{1, J}(I) \cdots a_{n, J}(I) \frac{e(J)}{M^{n}}$, by Theorem 4.2 , Then equality holds in (19).

The implications $(\mathrm{b}) \Rightarrow(\mathrm{c}) \Rightarrow(\mathrm{d})$ are obvious. Let us prove $(\mathrm{c}) \Rightarrow(\mathrm{b})$. Let $F=$ $\left(F_{1}, \ldots, F_{n}\right):\left(\mathbb{C}^{n}, 0\right) \rightarrow\left(\mathbb{C}^{n}, 0\right)$ be a complex analytic map such that $F$ is $J$-non-degenerate, $F$ is $J$-homogeneous and $\overline{I^{s}}=\overline{\left\langle F_{1}, \ldots, F_{n}\right\rangle}$, for some $s \in \mathbb{Z}_{\geqslant 1}$. Therefore, we have that $e\left(I^{s}\right)=e\left(F_{1}, \ldots, F_{n}\right)=A_{J}\left(F_{1}, \ldots, F_{n}\right)$. Let $d_{i}=\nu_{J}\left(F_{i}\right)$, for all $i=1, \ldots, n$. By reordering the components of $F$, we can assume that $d_{1} \leqslant \cdots \leqslant d_{n}$. Since $e\left(I^{s}\right)=s^{n} e(I)$ and we have already proved relation (19), we have

$$
\frac{d_{1} \cdots d_{n}}{s^{n} M^{n}}=\frac{e(I)}{e(J)} \leqslant \frac{a_{1, J}(I) \cdots a_{n, J}(I)}{M^{n}} .
$$

Then it suffices to prove that $a_{i, J}(I) \leqslant d_{i} / s$, for all $i=1, \ldots, n$. Thus 23 would imply that $a_{i, J}(I)=d_{i} / s$, for all $i=1, \ldots, n$, and hence (b) follows by considering the same map $F$ coming from assuming (c).

Let us fix an index $i \in\{1, \ldots, n\}$ and let $\Delta$ be a face of $\Gamma_{+}(J)$ of dimension $n-i$. The condition $\overline{I^{s}}=\overline{\left\langle F_{1}, \ldots, F_{n}\right\rangle}$ implies that $\Gamma_{+}\left(I^{s}\right)=\Gamma_{+}\left(F_{1}, \ldots, F_{n}\right)$. In particular,

$$
\begin{aligned}
\nu_{J}\left(\Gamma_{+}\left(I^{s}\right) \cap C(\Delta)\right) & =\nu_{J}\left(\Gamma_{+}\left(F_{1}, \ldots, F_{n}\right) \cap C(\Delta)\right) \\
& =\min \left\{d_{i}: \operatorname{supp}\left(\mathrm{p}_{\Delta}\left(F_{i}\right)\right) \neq 0\right\} .
\end{aligned}
$$

Since $F$ is $J$-non-degenerate and $\operatorname{dim} \mathcal{R}_{\Delta}=n-i+1$, there exist at least $n-i+1$ non-zero elements in the set $\left\{\mathrm{p}_{J, \Delta}\left(F_{1}\right), \ldots, \mathrm{p}_{J, \Delta}\left(F_{n}\right)\right\}$, by Theorem 4.2. This implies, by (24), that

$$
\nu_{J}\left(\Gamma_{+}\left(I^{s}\right) \cap C(\Delta)\right) \leqslant d_{n-(n-i+1)+1}=d_{i} .
$$

Therefore $s a_{i, J}(I)=a_{i, J}\left(I^{s}\right) \leqslant d_{i}$, for all $i=1, \ldots, n$. That is, $a_{i, J}(I) \leqslant d_{i} / s$, for all $i=1, \ldots, n$.

Finally, let us prove $(\mathrm{d}) \Rightarrow(\mathrm{c})$. Let $g=\left(g_{1}, \ldots, g_{n}\right)$ be a $J$-non-degenerate map and let $s \geqslant 1$ such that $\overline{I^{s}}=\overline{\left\langle g_{1}, \ldots, g_{n}\right\rangle}$. Let $F_{i}=\mathrm{p}_{J}\left(g_{i}\right)$, for all $i=1, \ldots, n$, and let $F=$ $\left(F_{1}, \ldots, F_{n}\right)$. We observe that $F$ is $J$-non-degenerate and $J$-homogeneous. In particular, $e\left(I^{s}\right)=e\left(g_{1}, \ldots, g_{n}\right)=e\left(F_{1}, \ldots, F_{n}\right)$. Moreover $\Gamma_{+}\left(F_{i}\right) \subseteq \Gamma_{+}\left(g_{i}\right) \subseteq \Gamma_{+}\left(I^{s}\right)$, for all $i=$ $1, \ldots, n$. Since $I$ is a monomial ideal, the integral closure of $I^{s}$ is also a monomial ideal. Therefore $\overline{\left\langle F_{1}, \ldots, F_{n}\right\rangle} \subseteq \overline{I^{s}}$. Then we obtain the equality $\overline{\left\langle F_{1}, \ldots, F_{n}\right\rangle}=\overline{I^{s}}$, by the Rees' multiplicity theorem, and thus item (c) follows. 
Remark 5.6. As we showed in the proof of Theorem 5.5, if equality holds in (19), then we can take $s=c_{J}(I) M_{J}$ in items (b), (c) and (d) of Theorem 5.5.

Let $I$ be a monomial ideal of $\mathcal{O}_{n}$ of finite colength. Let us observe that $c_{\mathbf{m}_{n}}(I)=1$ and $M_{\mathbf{m}_{n}}=1$. Hence, by Theorem 5.5 and $(16)$ we obtain that $e(I) \leqslant a_{1}(I) \cdots a_{n}(I)$ and equality holds if and only if there exist polynomials $F_{1}, \ldots, F_{n} \in \mathbb{C}\left[x_{1}, \ldots, x_{n}\right]$ such that $F_{i}$ is homogeneous of degree $a_{i}(I)$, for all $i \in\{1, \ldots, n\}$, and $\bar{I}=\overline{\left\langle F_{1}, \ldots, F_{n}\right\rangle}$. Hence, by using different techniques, we deduce Theorem 3.5 of [4] as the case $J=\mathbf{m}_{n}$ of Theorem 5.5 .

Let $I$ and $J$ be monomial ideals of $\mathcal{O}_{n}$ of finite colength. Then we define

$$
\mathrm{C}_{J}(I)=\frac{a_{1, J}(I) \cdots a_{n, J}(I)}{M_{J}^{n}} .
$$

In (25) we will see that $1 \leqslant \mathrm{C}_{I}(J) \mathrm{C}_{J}(I)$, where equality does not hold in general, as we show in Example 5.8 .

Corollary 5.7. Let $I, J \subseteq \mathcal{O}_{n}$ be monomial ideals of $\mathcal{O}_{n}$ of finite colength. Then

$$
\frac{1}{\mathrm{C}_{I}(J)} \leqslant \frac{e(I)}{e(J)} \leqslant \mathrm{C}_{J}(I)
$$

and the following conditions are equivalent:

(a) equality holds in some part of (25);

(b) equality holds in both parts of (25);

(c) $\mathrm{C}_{I}(J) \mathrm{C}_{J}(I)=1$.

Proof. Relation (25) is an immediate application of relation (19), in Theorem 5.5.

Let us see the implication (a) $\Rightarrow(\mathrm{b})$. Let us assume that $e(I) / e(J)=\mathrm{C}_{J}(I)$. By Theorem 5.5, there exists a $J$-non-degenerate map $g=\left(g_{1}, \ldots, g_{n}\right):\left(\mathbb{C}^{n}, 0\right) \rightarrow\left(\mathbb{C}^{n}, 0\right)$ and an integer $s \geqslant 1$ such that $\overline{I^{s}}=\overline{\left\langle g_{1}, \ldots, g_{n}\right\rangle}$. By Proposition 4.6 , there exist $r, a_{1}, \ldots, a_{n} \in \mathbb{Z}_{\geqslant 1}$ such that $\overline{J^{r}}=\overline{\left\langle g_{1}^{a_{1}}, \ldots, g_{n}^{a_{n}}\right\rangle}$.

Since $I$ is a monomial ideal, then $\overline{I^{s}}$ is also a monomial ideal and hence the equality $\overline{I^{s}}=\overline{\left\langle g_{1}, \ldots, g_{n}\right\rangle}$ implies that $\left\langle g_{1}, \ldots, g_{n}\right\rangle$ is a Newton non-degenerate ideal whose Newton polyhedron is equal to $s \Gamma_{+}(I)$, by [7, Corollary 2.6] or [2, Proposition 3.6]. Then $\left(g_{1}^{\alpha_{1}}, \ldots, g_{n}^{\alpha_{n}}\right)$ is $I$-non-degenerate, for any $\alpha_{1}, \ldots, \alpha_{n} \in \mathbb{Z}_{\geqslant 1}$. In particular, $\left(g_{1}^{a_{1}}, \ldots, g_{n}^{a_{n}}\right)$ is $I$-non-degenerate. Joining this fact with the equality $\overline{J^{r}}=\overline{\left\langle g_{1}^{a_{1}}, \ldots, g_{n}^{a_{n}}\right\rangle}$, we conclude that $e(J) / e(I)=\mathrm{C}_{I}(J)$, by Theorem 5.5. Following an analogous argument, we obtain that if $e(J) / e(I)=\mathrm{C}_{I}(J)$ then $e(I) / e(J)=\mathrm{C}_{J}(I)$.

The implication (b) $\Rightarrow$ (a) is trivial. The equivalence between (b) and (c) is an immediate consequence of 25).

If $I, J$ is any pair of monomial ideals in $\mathcal{O}_{n}$ of finite colength, then we write $I \sim J$ if $\mathrm{C}_{I}(J) \mathrm{C}_{J}(I)=1$. Let us observe that $\sim$ is a reflexive and symmetric relation. However $\sim$ is not a transitive relation, as the following example shows.

Example 5.8. Let us consider the ideals of $\mathcal{O}_{2}$ given by $I=\left\langle x y, x^{5}, y^{5}\right\rangle, J=\mathbf{m}_{2}=\langle x, y\rangle$ and $K=\left\langle x, y^{2}\right\rangle$. We observe that $\mathbf{a}_{I}(J)=\left(1, \frac{5}{2}\right), \mathbf{a}_{J}(K)=(1,2)$ and $\mathbf{a}_{I}(K)=\left(2, \frac{10}{3}\right)$. 
Moreover $M_{I}=5, M_{J}=1, M_{K}=2, e(I)=10, e(J)=1$ and $e(K)=2$. Therefore $\mathrm{C}_{I}(J) \mathrm{C}_{J}(I)=1$ and $\mathrm{C}_{J}(K) \mathrm{C}_{K}(J)=1$. However $\mathrm{C}_{I}(K)=\frac{4}{15}$ and $\mathrm{C}_{K}(I)=\frac{15}{2}$ and hence $1<\mathrm{C}_{I}(K) \mathrm{C}_{K}(I)=2$.

\section{REFERENCES}

[1] V.I. Arnold, S. Gusein-Zade and A. Varchenko, Singularities of differentiable maps. Volume I: The classification of critical points, caustics and wave fronts, Monogr. Math. Vol. 82 (1985), Birkhäuser. 1

[2] C. Bivià-Ausina, Joint reductions of monomial ideals and multiplicity of complex analytic maps, Math. Res. Lett. 15, No. 2 (2008), 389-407. 2.1, 2.1, 2.1, 2.2, 2.2, 4.2, 5

[3] C. Bivià-Ausina, Local Łojasiewicz exponents, Milnor numbers and mixed multiplicities of ideals, Math. Z. 262, No. 2 (2009), 389-409. 2.1, 5.2

[4] C. Bivià-Ausina, Multiplicity and Eojasiewicz exponent of generic linear sections of monomial ideals, Bull. Aust. Math. Soc. 91, No. 2, 191-201 (2015). 1. 5.2, 5.6

[5] C. Bivià-Ausina and S. Encinas, Lojasiewicz exponent of families of ideals, Rees mixed multiplicities and Newton filtrations, Rev. Mat. Complut. 26 (2013), No. 2, 773-798. 2.1. 4.1. 5.2

[6] C. Bivià-Ausina and T. Fukui, Mixed Eojasiewicz exponents and log canonical thresholds of ideals, J. Pure and Appl. Algebra 220 (2016), No. 1, 223-245. 5.2

[7] C. Bivià-Ausina, T. Fukui and M.J. Saia, Newton graded algebras and the codimension of non-degenerate ideals, Math. Proc. Cambridge Philos. Soc. 133 (2002), 55-75. 1, 1, 2.2, 4.1, 4.1, 4.1, 5

[8] E.M. Chirka, Complex analytic sets, Translated from the Russian by R. A. M. Hoksbergen. Mathematics and its Applications (Soviet Series), 46. Kluwer Academic Publishers Group, Dordrecht, 1989. 1

[9] J.P. D'Angelo, Several complex variables and the geometry of real hypersurfaces, Studies in Advanced Mathematics. CRC Press, Boca Raton, FL, 1993. 1

[10] M. Herrmann, S. Ikeda and U. Orbanz, Equimultiplicity and Blowing Up. An Algebraic Study with an Appendix by B. Moonen, Springer, Berlin (1988) 1, 2.1, 2.1, 5

[11] M. Hickel, Fonction asymptotique de Samuel des sections hyperplanes et multiplicité, J. Pure Appl. Algebra 214 (2010), No. 5, 634-645. 1. 5.2

[12] C. Huneke and I. Swanson, Integral Closure of Ideals, Rings, and Modules, London Math. Soc. Lecture Note Series 336 (2006), Cambridge University Press. 1, 1, 2.1, 2.1, 2.1, 2.1, 4.1, 4.1, 5

[13] A.G. Kouchnirenko, Polyèdres de Newton et nombres de Milnor, Invent. Math. 32 (1976), 1-31. 2.2 4.1

[14] D. Rees, Generalizations of reductions and mixed multiplicities, J. London Math. Soc. (2) 29 (1984), 397-414. 1. 2.1, 2.1.

[15] D. Rees and J. Sally, General elements and joint reductions, Mich. Math. J. 35, No. 2 (1988), 241-254. 1. 2.1

[16] P.C. Roberts, Multiplicities and Chern classes in local algebra., Cambridge Tracts in Mathematics, 133. Cambridge University Press, Cambridge, 1998. 2.1

[17] M.J. Saia, The integral closure of ideals and the Newton filtration, J. Algebraic Geom. 5 (1996), 1-11. 2.2

[18] I. Swanson, Mixed multiplicities, joint reductions and quasi-unmixed local rings, J. London Math. Soc. (2) 48, No. 1 (1993), 1-14. 2.1, 4.1

[19] B. Teissier, Cycles évanescents, sections planes et conditions of Whitney, Singularités à Cargèse, Astérisque, no. 7-8 (1973), 285-362.2.1.2.1

[20] B. Teissier, Variétés Polaires II. Multiplicites polaires, sections planes et conditions de Whitney, Algebraic geometry (La Rábida, 1981), 314-491, Lecture Notes in Math. 961, Springer, Berlin, 1982. 4.9 
[21] B. Teissier, Monômes, volumes et multiplicités, Introduction à la théorie des singularités, II, Travaux en Cours, 37, Hermann, 1988, 127-141. 1

[22] B. Teissier, Monomial ideals, binomial ideals, polynomial ideals, Trends in commutative algebra, Math. Sci. Res. Inst. Publ., 51, 211-246. Cambridge Univ. Press, Cambridge, 2004. 1. 4.1

[23] W. Vasconcelos, Integral closure. Rees algebras, multiplicities, algorithms. Springer Monographs in Mathematics. Springer-Verlag, Berlin, 2005. 1 ,2.1

[24] C.T.C. Wall, Newton polytopes and non-degeneracy, J. Reine Angew Math. 509 (1999), 1-19. 2.2

Institut Universitari de Matemàtica Pura i Aplicada, Universitat Politècnica de València, Camí de Vera, s/n, 46022 València, Spain

E-mail address: carbivia@mat.upv.es 